\title{
Young radio-loud gamma-ray pulsar light curve fitting
}

\author{
J. Pétri ${ }^{1}$ and D. Mitra ${ }^{2,3}$ \\ 1 Université de Strasbourg, CNRS, Observatoire astronomique de Strasbourg, UMR 7550, 67000 Strasbourg, France \\ e-mail: jerome.petri@astro.unistra.fr \\ 2 National Centre for Radio Astrophysics, Tata Institute for Fundamental Research, Post Bag 3, Ganeshkhind, Pune 411007, India \\ 3 Janusz Gil Institute of Astronomy, University of Zielona Góra, ul. Szafrana 2, 65-516 Zielona Góra, Poland
}

Received 8 May 2021 / Accepted 13 July 2021

\begin{abstract}
Context. Since the launch of the Fermi gamma-ray telescope, several hundred radio-loud gamma-ray pulsars have been detected, many belonging to millisecond pulsars but some belonging to the young pulsar population with spin periods longer than $30 \mathrm{~ms}$.

Aims. Observing simultaneously pulsed radio and gamma-ray emission from these stars helps to constrain the geometry and radiation mechanisms within their magnetosphere and to localize the multiple photon production sites. In this paper we fit the time-aligned gamma-ray light curves of young radio-loud gamma-ray pulsars. We assume a dipole force-free magnetosphere where radio photons emanate from high altitudes above the polar caps and gamma rays originate from outside the light cylinder, within the striped wind current sheet.

Methods. We computed a full atlas of radio and gamma-ray pulse profiles depending on the magnetic axis obliquity and line-of-sight inclination with respect to the neutron star rotation axis. By applying a $\chi^{2}$ fitting technique, we were able to pin down accurately the magnetosphere geometry. Further constraints were obtained from radio polarization measurement following the rotating vector model, including aberration and retardation effects.

Results. We find a good agreement between our model and the time-aligned single- or double-peaked gamma-ray pulsar observations. We deduce the magnetic inclination angle and the observer line of sight with respect to the rotation axis within a small error bar. The distinction between radio-loud or radio-quiet gamma-ray pulsars or only radio pulsars can entirely be related to the geometry of the associated emitting regions.

Conclusions. The high-altitude polar cap model combined with the striped wind represents a minimalistic approach able to reproduce a wealth of gamma-ray pulse profiles for young radio pulsars. Based on self-consistent force-free simulations, it gives a full geometrical picture of the emission properties without resorting to detailed knowledge of the individual particle dynamics and energetics.
\end{abstract}

Key words. magnetic fields - polarization - magnetohydrodynamics (MHD) - radiation mechanisms: general - stars: neutron gamma rays: stars

\section{Introduction}

The observed pulsed emission properties of pulsars in the radio and high-energy bands, like their light curves and spectra, are very sensitive to their global geometry. This geometry is defined by their electromagnetic field topology and the angles, on the one hand between the rotation axis and the magnetic dipole axis, and on the other hand between the rotation axis and the line of sight. A good first guess about the electromagnetic field was given by the now comprehensive simulations of dipole force-free magnetospheres for aligned rotators (Contopoulos et al. 1999; Komissarov 2006; Parfrey et al. 2012; Cao et al. 2016a) and oblique rotators (Spitkovsky 2006; Pétri 2012) and (Kalapotharakos et al. 2012). More detailed models include some dissipation through resistivity, like those done in Li et al. (2012), Kalapotharakos et al. (2017) and Cao et al. (2016b). Even kinetic simulations are available (Cerutti et al. 2015). However, a force-free fluid approach already suffices to construct realistic radio and gamma-ray pulse profiles, furnishing severe constraints on the underlying geometry.

Several works in the past indeed showed that a simultaneous radio and gamma-ray light curve fitting is valuable to pin down the geometry. For instance, Pétri (2011) showed that in the framework of a force-free split-monopole solution, simple analytical expressions for the radio time lag and the gammaray peak separation can be derived. Meanwhile, Seyffert et al. (2011) used an emission model for gamma rays (relying on outer gaps or two-pole caustics) and the constraints from radio polarization to deduce the geometry of several pulsars, soon after the publication of the first Fermi gamma-ray pulsar catalogue (Abdo et al. 2010). Pierbattista et al. (2015) performed a comprehensive analysis of light curve modelling of young gammaray pulsars assuming different geometries like polar cap, slot gap, outer gap, and one-pole caustic but did not include the striped wind. They also pointed out the importance of joint radio- $\gamma$-ray fit to constrain the geometry. Some refinements of this approach are due to Pierbattista et al. (2016). A complete atlas of gamma-ray pulse profiles for several magnetospheric models, summarizing the pulse properties and merits of each of them can be found in Watters et al. (2009).

Other useful constraints on the emission sites come from detailed radio polarization observations. However, if they come from millisecond pulsars, these polarization data are difficult to interpret because of the presence of strong non-dipolar fields at the photon production sites. Nevertheless, Benli et al. (2021) were able to put constraints on some of these millisecond pulsars by fitting the time-aligned gamma-ray light curves without resorting to accurate radio pulse profile modelling. 
Their model is based on accurate dipole force-free magnetosphere simulations. Fortunately, the situation is drastically better for young radio-loud gamma-ray pulsars. Thanks to radio polarization measurements following the rotating vector model (Radhakrishnan \& Cooke 1969), aberration and/or retardation effects (Blaskiewicz et al. 1991) help to localize the altitude of radio emission which is about $5 \%$ of the light cylinder radius for the sample studied in Mitra (2017). In this paper we apply the fitting procedure used by Benli et al. (2021) to young pulsars for which the radio emission height is better constrained and the polarization data reasonably follow the rotating vector model, thus relying on a pure dipole field with high confidence.

In this paper we re-explore the work done by Pétri (2011) by using a realistic dipole force-free magnetosphere solution extracted from our numerical simulations. The paper is organized as follows. Section 2 summarizes the radio observations using polarization data to constrain emission heights and the geometry. Section 3 summarizes the emission properties of the current sheet within the force-free split monopole framework of Michel (1973) and Bogovalov (1999). Time-aligned radio and gamma-ray light curves are computed for geometric configurations and summarized in several sky maps. Then we generalize this approach to the more realistic dipole field, smoothly joining the stellar surface to the striped wind and referred to as the dipole force-free magnetosphere. Section 4 shows the results of our fitting procedure for a good sample of young pulsars, constraining their geometry. We draw our conclusions in Sect. 5.

\section{Radio observations}

Pulsars are broadband emitters and various frequencies emanating from different parts of the pulsar magnetosphere. The location of the broadband emission is best constrained for the pulsed radio emission, which is thought to arise from regions near the neutron star polar cap, and the $\gamma$ rays, which are thought to arise near the light cylinder. Magnetospheric simulations of pulsars assume a star-centred dipolar magnetic field configuration, and are restricted to fast rotating pulsars (roughly $10 \mathrm{~ms}$ ) due to limitation in numerical resolution and computation time. Thus, to compare simulation results with observations, ideally it is best to use millisecond pulsars (MSP) to identify emission zones in pulsars where the magnetic field is dipolar. Similar studies have been done (see e.g., Benli et al. 2021); however, in the case of MSPs it is difficult to constrain both the location and magnetic field structure in the radio emission region, and as we discuss below the young pulsar population can be used to obtain significantly better constraints.

Radio polarization observations are particularly useful in this regard since the polarization properties can be used to find both the location of the emission site and its magnetic field geometry. The polarization position angle (PPA) of the pulsar linear polarization shows a characteristic S-shape across the pulse profile. The PPA traverse can be interpreted in terms of the rotating vector model (RVM, Radhakrishnan \& Cooke 1969), which states that the PPA traverse reflects the change in the diverging dipolar magnetic field line planes as the pulse profile sweeps past the observer. According to the RVM, the PPA as a function of the pulse phase $\phi$ is given by

$$
\Psi(\phi)=\Psi_{\circ}+\arctan \left(\frac{\sin \alpha \sin \left(\phi-\phi_{\circ}\right)}{\sin \zeta \cos \alpha-\sin \alpha \cos \zeta \cos \left(\phi-\phi_{\circ}\right)}\right),
$$

where $\alpha$ is the angle between the rotation axis and the dipole magnetic axis, $\beta$ is the angle between the magnetic axis and the observer line of sight, and $\zeta=\alpha+\beta$ is the angle between the rotation axis and the observer line of sight; $\Psi$ 。 corresponds to the steepest gradient point of the RVM which occurs at the longitude $\phi_{\text {o, }}$ such that

$$
\left.\frac{\mathrm{d} \Psi}{\mathrm{d} \phi}\right|_{\phi_{\circ}}=\frac{\sin \alpha}{\sin \beta}
$$

While in principle fitting Eq. (1) to the PPA traverse in pulsars can constrain the magnetic field geometry $\alpha$ and $\beta$, in practice these parameters are highly correlated, so they remain unconstrained (see e.g., Everett \& Weisberg 2001). Nonetheless a good fit of the observed PPA to the RVM indicates that the pulsar radio emission arises from regions of dipolar magnetic field regions.

In this study we focus on 31 young pulsars with periods longer that $50 \mathrm{msec}$ (see Table 1). Young pulsars are generally known to be highly polarized, and their PPA are often consistent with the RVM. Of the 31 pulsars in our sample, we could obtain archival polarization data for 21 pulsars at $1.4 \mathrm{GHz}$ from Johnston \& Kerr (2018) and Theureau et al. (2011). Furthermore, we could reliably fit the RVM given by Eq. (1) for 17 pulsars. The reduced $\chi^{2}$ values for the fits corresponding to the $\alpha$ and $\zeta=\alpha+\beta$ values given in Table 2 are given in Col. 7 of Table 1 , and in most cases they indicate that the RVM is a good fit to the PPA traverse. The large reduced $\chi^{2}$ value for PSR J0908-4913 and J0835-4510 mostly occurs due to certain abrupt changes in the PPA traverse towards the edge of the profiles. Such changes in pulsar average PPA traverse can occur due to orthogonal polarization moding or emission across the profile arising due to a range of heights (see Mitra \& Seiradakis 2004; Mitra et al. 2007). However, for both these pulsars the overall PPA traverse is consistent with the RVM when these kinky regions are excluded.

For some pulsars mentioned as 'No RVM', it was not possible to constrain the RVM either due to scattering (PSR J0248+6021, PSR J1019-5749, J1730-3350), low polarization (PSR J1509-5830), or extremely flat PPA traverse (PSR J1016-5857, J1028-5819). For several pulsars in our sample, RVM fits and $\chi^{2}$ contours have been reported by Rookyard et al. (2015), Weltevrede \& Wright (2009) and Kramer \& Johnston (2008), and our results are in good agreement with these earlier studies. For the cases where RVM fits were possible, it can be concluded that the radio emission arises from regions of dipolar magnetic field lines. While it is desirable to model the polarization for all the pulsars in our sample, in the absence of such data we currently assume that this conclusion is applicable for our whole sample of young pulsars.

Next we turn our attention to finding the location of the radio emission region. It has been suggested by Blaskiewicz et al. (1991) that due to rotation of the pulsar a delay $(\Delta \phi)$ is introduced between the centre of the pulse profile and the steepest gradient point of the PPA, as a result of the aberrationretardation $(\mathrm{A}-\mathrm{R})$ effect. This delay, in the linear approximation, is related to the radio emission height $h$ from the stellar surface and the pulsar period $P$ as $\Delta \phi=1440 h / c P$ (deg), where $c$ is the velocity of light. The radio emission heights using the A-R delay has been estimated in a large sample of pulsars by several studies (e.g., Blaskiewicz et al. 1991; Mitra \& Li 2004; Weltevrede \& Johnston 2008). The emission height as a function of pulsar period calculated using the A-R method is found to originate about $500 \mathrm{~km}$ above the neutron star surface (see Mitra 2017). As the emission height appears to be constant over a wide period range, the value of $\Delta \phi$ is expected to be much larger 
Table 1. Radio profile of Young radio-loud gamma-ray pulsars extracted for the Fermi second pulsar catalogue.

\begin{tabular}{|c|c|c|c|c|c|c|c|c|c|}
\hline $\begin{array}{l}\text { PSR } \\
(\mathrm{J} 2000)\end{array}$ & $\begin{array}{c}P \\
(\mathrm{~ms}) \\
\end{array}$ & $\delta$ & $\Delta$ & $\begin{array}{c}\phi_{1} \\
(\mathrm{deg})\end{array}$ & $\begin{array}{c}\phi_{\mathrm{t}} \\
(\mathrm{deg})\end{array}$ & $\chi^{2}$ & $\begin{array}{c}\phi_{\circ} \\
(\mathrm{deg})\end{array}$ & $\begin{array}{c}\Delta \phi \\
(\mathrm{deg})\end{array}$ & $\begin{array}{c}\text { Height }(h) \\
(\mathrm{km})\end{array}$ \\
\hline $\mathrm{J} 0248+6021$ & 217 & $0.336 \pm 0.017$ & - & $-7.7 \pm 0.2$ & $65.4 \pm 0.2$ & & NO RVM & & \\
\hline $\mathrm{J} 0631+1036$ & 288 & $0.497 \pm 0.022$ & - & $-7.7 \pm 0.3$ & $14.4 \pm 0.3$ & 1.16 & $14 \pm 2$ & $11 \pm 2$ & $660 \pm 120$ \\
\hline J0659+1414 & 385 & $0.224 \pm 0.010$ & - & $-18.9 \pm 0.4$ & $15.5 \pm 0.4$ & 2.05 & $22 \pm 7$ & $23 \pm 7$ & $1884 \pm 561$ \\
\hline J0742-2822 & 167 & $0.627 \pm 0.005$ & - & $-12.6 \pm 0.3$ & $17.5 \pm 0.3$ & 42.7 & $8 \pm 2$ & $6 \pm 2$ & $208 \pm 69$ \\
\hline J0835-4510 & 89 & $0.129 \pm 0.001$ & $0.433 \pm 0.001$ & $-38.6 \pm 0.2$ & $37.6 \pm 0.2$ & 3245 & $4.3 \pm 0.5$ & $5 \pm 1$ & $93 \pm 20$ \\
\hline J0908-4913 ${ }^{\dagger}$ & 107 & $0.102 \pm 0.005$ & $0.501 \pm 0.006$ & $-15.8 \pm 0.3$ & $12.3 \pm 0.3$ & 1466 & $3.4 \pm 1$ & $5 \pm 1$ & $111 \pm 22$ \\
\hline J1016-5857 & 107 & $0.143 \pm 0.003$ & $0.423 \pm 0.004$ & $-19.3 \pm 0.3$ & $10.2 \pm 0.3$ & & NO RVM & & \\
\hline J1019-5749 & 162 & $0.482 \pm 0.010$ & - & $-10.5 \pm 0.3$ & $52.7 \pm 0.3$ & & NO RVM & & \\
\hline J1028-5819 & 91 & $0.195 \pm 0.001$ & $0.475 \pm 0.001$ & $-1.8 \pm 0.4$ & $0.4 \pm 0.3$ & & NO RVM & & \\
\hline $\mathrm{J} 1048-5832$ & 124 & $0.125 \pm 0.001$ & $0.426 \pm 0.001$ & $-16.2 \pm 0.3$ & $20.4 \pm 0.3$ & 6.6 & $3.5 \pm 1$ & $1 \pm 1$ & \\
\hline $\mathrm{J} 1057-5226^{\dagger}$ & 197 & $0.304 \pm 0.003$ & $0.307 \pm 0.004$ & $-13.7 \pm 0.3$ & $23.2 \pm 0.3$ & 11.09 & $0.0 \pm 18$ & $-4 \pm 18$ & \\
\hline J1119-6127 & 408 & $0.285 \pm 0.015$ & $0.204 \pm 0.020$ & $-15.1 \pm 0.3$ & $15.8 \pm 0.3$ & 1.5 & $26.0 \pm 10$ & $25 \pm 10$ & $2125 \pm 850$ \\
\hline J1357-6429 & 166 & $0.359 \pm 0.028$ & - & $-15.8 \pm 0.3$ & $16.2 \pm 0.3$ & 1.33 & $2.5 \pm 100$ & $2 \pm 100$ & \\
\hline J1420-6048 & 68 & $0.196 \pm 0.011$ & $0.312 \pm 0.015$ & $-39.7 \pm 0.3$ & $12.3 \pm 0.3$ & 1.9 & $-10.7 \pm 3$ & $3 \pm 3$ & \\
\hline J1509-5850 & 89 & $0.271 \pm 0.011$ & $0.264 \pm 0.013$ & $-7.1 \pm 0.3$ & $8.1 \pm 0.3$ & & NO RVM & & \\
\hline J1648-4611 & 165 & $0.261 \pm 0.010$ & $0.298 \pm 0.082$ & $-14.7 \pm 0.3$ & $9.1 \pm 0.3$ & 1.6 & $-9.6 \pm 10$ & $-6 \pm 10$ & \\
\hline $\mathrm{J} 1702-4128$ & 182 & $0.397 \pm 0.038$ & - & $-11.9 \pm 0.3$ & $16.5 \pm 0.3$ & 1.4 & $-17.9 \pm 30$ & $-20 \pm 30$ & \\
\hline J1709-4429 & 102 & $0.239 \pm 0.001$ & $0.244 \pm 0.002$ & $-30.9 \pm 0.3$ & $29.5 \pm 0.3$ & 1.07 & $10.3 \pm 3$ & $11 \pm 3$ & $233 \pm 64$ \\
\hline J1718-3825 & 75 & $0.397 \pm 0.009$ & - & $-10.9 \pm 0.3$ & $38.6 \pm 0.3$ & 1.26 & $21.4 \pm 5$ & $9 \pm 5$ & $140 \pm 78$ \\
\hline $\mathrm{J} 1730-3350$ & 139 & $0.128 \pm 0.007$ & $0.419 \pm 0.007$ & $-11.6 \pm 0.3$ & $50.8 \pm 0.3$ & & NO RVM & & \\
\hline $\mathrm{J} 1747-2958$ & 99 & $0.181 \pm 0.003$ & $0.392 \pm 0.005$ & NRP & & & & & \\
\hline J1801-2451 & 125 & $0.060 \pm 0.005$ & $0.496 \pm 0.020$ & $-10.4 \pm 0.3$ & $10.9 \pm 0.3$ & 0.87 & $-8.6 \pm 6$ & $-9 \pm 6$ & \\
\hline J1835-1106 & 166 & $0.139 \pm 0.006$ & $0.421 \pm 0.011$ & $-11.2 \pm 0.3$ & $11.2 \pm 0.3$ & 1.37 & $4.2 \pm 5$ & $4 \pm 5$ & \\
\hline $\mathrm{J} 1907+0602$ & 107 & $0.209 \pm 0.003$ & $0.389 \pm 0.004$ & NRP & & & & & \\
\hline $\mathrm{J} 1952+3252$ & 39 & $0.161 \pm 0.002$ & $0.478 \pm 0.003$ & NRP & & & & & \\
\hline $\mathrm{J} 2021+3651$ & 104 & $0.132 \pm 0.001$ & $0.478 \pm 0.001$ & NRP & & & & & \\
\hline $\mathrm{J} 2030+3641$ & 200 & $0.269 \pm 0.010$ & $0.309 \pm 0.014$ & NRP & & & & & \\
\hline $\mathrm{J} 2032+4127$ & 143 & $0.099 \pm 0.001$ & $0.516 \pm 0.001$ & NRP & & & & & \\
\hline $\mathrm{J} 2043+2740$ & 96 & $0.132 \pm 0.007$ & $0.432 \pm 0.010$ & NRP & & & & & \\
\hline $\mathrm{J} 2229+6114$ & 52 & $0.187 \pm 0.007$ & $0.299 \pm 0.008$ & NRP & & & & & \\
\hline $\mathrm{J} 2240+5832$ & 140 & $0.118 \pm 0.014$ & $0.476 \pm 0.014$ & $-8.6 \pm 0.2$ & $7.4 \pm 0.2$ & 1.93 & $3.7 \pm 10$ & $4 \pm 10$ & \\
\hline
\end{tabular}

Notes. The data for PSR J2240+5832 and PSR J0248+6021 are from Theureau et al. (2011). The rest of the data are from Johnston \& Kerr (2018), and the abbreviation 'NRP' stands for cases where no radio profile was available for analysis. The abbreviation 'NO RVM' corresponds to cases where the RVM fit was not possible for the data. The pulsars flagged with superscript $\dagger$ are interpulsars, where the $\phi_{\circ}, \Delta \phi$, and $h$ in the table is estimated for the region below the main pulse.

in younger pulsars than older pulsars. As a result more robust radio emission heights can be obtained in the younger pulsar population.

However, there are certain limitations in applying the A-R delay method for emission height estimates. In order to estimate $\Delta \phi$ the longitude at the leading $\left(\phi_{1}\right)$ and trailing $\left(\phi_{\mathrm{t}}\right)$ edge of the profile is obtained, as well as the longitude corresponding to the steepest gradient point $\left(\phi_{\circ}\right)$ of the PPA traverse from RVM fits. The A-R effect predicts a positive $\Delta \phi$, where $\Delta \phi=\phi_{\circ}-\left(\phi_{\mathrm{t}}-\right.$ $\left.\phi_{1}\right) / 2$. It has been noted in several studies (e.g., Mitra \& Li 2004; Weltevrede \& Johnston 2008) that there are pulsars for which $\Delta \phi$ is negative, and hence do not reflect the A-R effect. The limitations stem from the fact that the A-R method requires $\phi_{1}$ and $\phi_{\mathrm{t}}$ to correspond to the last open magnetic field line which is symmetrically placed with respect to the magnetic axis. However, $\phi_{1}$ and $\phi_{\mathrm{t}}$ are measured at a few times above the noise level at the edge of the profile, and this can lead to errors due to weak emission near the profile edges. Mitra \& Rankin (2011) showed that single pulse studies can be beneficial to estimate profile edges, as some single pulses (where the edge emission is brighter and more prominent) can be significantly stronger than the average profile. In addition, the $\phi_{\circ}$ measurements can also be affected due to the presence of orthogonal polarization modes, and single pulses can be used to model the RVM significantly better by disentangling the orthogonal polarization moding effects.

Our sample pulsars, however, has only average profile data, and in Table 1 we give the $\phi_{1}$ and $\phi_{\mathrm{t}}$ measured at 5 times the rms level measured in the off-pulse region. The fiducial points $\phi_{\circ}$ obtained by the RVM fits are also given in the table. Using these values we estimated the emission heights $h$ as shown in Table 1. A reliable $h$ value could be estimated for eight cases, and was found to lie below $10 \%$ of the light cylinder radius, which we assume to be a good estimate for our pulsar sample.

\section{Split monopole versus dipole magnetosphere}

Computing multi-wavelength light curves relies on some magnetosphere models. In this paper we exclusively consider force-free models based on either a split-monopole or a dipole magnetic field. Before showing the results of the dipole magnetosphere fitting the observations, it is instructive to compare the split-monopole expectations to the more realistic dipole field. 
Table 2. Best fit values for the geometry of each pulsar according to the analysis of their gamma-ray light curves.

\begin{tabular}{|c|c|c|c|}
\hline PSR & $\alpha\left(\right.$ in $\left.^{\circ}\right)$ & $\zeta\left(\right.$ in $\left.^{\circ}\right)$ & $\phi_{\mathrm{s}}$ \\
\hline $\mathrm{J} 0248+6021$ & 30 & 42 & -0.07 \\
\hline $\mathrm{J} 0631+1036^{*}$ & 40 & 36 & 0.01 \\
\hline $\mathrm{J} 0659+1414^{*}$ & 45 & 32 & -0.23 \\
\hline J0742-2822* & 140 & 136 & 0.16 \\
\hline J0835-4510* & 65 & 58 & -0.1 \\
\hline J0908-4913* & 95 & 92 & -0.08 \\
\hline J1016-5857 & 40 & 64 & -0.02 \\
\hline J1019-5749 & 30 & 42 & 0.07 \\
\hline J1028-5819 & 75 & 64 & -0.01 \\
\hline $\mathrm{J} 1048-5832^{*}$ & 60 & 68 & -0.12 \\
\hline $\mathrm{J} 1057-5226^{*}$ & 25 & 44 & -0.01 \\
\hline $\mathrm{J} 1119-6127^{*}$ & 60 & 40 & -0.06 \\
\hline J1357-6429* & 20 & 34 & -0.09 \\
\hline J1420-6048* & 45 & 56 & -0.08 \\
\hline J1509-5850 & 40 & 24 & -0.06 \\
\hline J1648-4611* & 60 & 42 & -0.05 \\
\hline $\mathrm{J} 1702-4128^{*}$ & 155 & 148 & -0.05 \\
\hline J1709-4429* & 40 & 56 & -0.1 \\
\hline $\mathrm{J} 1718-3825^{*}$ & 30 & 38 & -0.06 \\
\hline $\mathrm{J} 1730-3350$ & 20 & 36 & -0.05 \\
\hline $\mathrm{J} 1747-2958$ & 45 & 68 & -0.07 \\
\hline $\mathrm{J} 1801-2451^{*}$ & 85 & 72 & -0.12 \\
\hline $\mathrm{J} 1835-1106^{*}$ & 30 & 36 & 0.03 \\
\hline $\mathrm{J} 1907+0602$ & 45 & 68 & -0.04 \\
\hline $\mathrm{J} 1952+3252$ & 60 & 84 & -0.06 \\
\hline $\mathbf{J} 2021+3651$ & 60 & 80 & -0.09 \\
\hline $\mathrm{J} 2030+3641$ & 20 & 42 & -0.07 \\
\hline $\mathbf{J} 2032+4127$ & 85 & 72 & -0.08 \\
\hline $\mathbf{J} 2043+2740$ & 45 & 68 & -0.06 \\
\hline $\mathrm{J} 2229+6114$ & 35 & 44 & -0.03 \\
\hline $\mathrm{J} 2240+5832^{*}$ & 60 & 80 & -0.09 \\
\hline
\end{tabular}

Notes. Pulsars flagged with superscript $*$ are those that have radio polarization RVM fits (see Table 1) using the $\alpha$ and $\zeta$ values given above (except for PSR J1057-5226, as discussed in Sect. 4.3).

\subsection{Split monopole}

The split monopole is a simple but elegant exact 3D force-free solution of a neutron star magnetosphere at large distances $r \gg$ $r_{\mathrm{L}}$, well outside the light cylinder, in the wind zone. We note however that the magnetic field strength decreases only like a monopole, that is with $B \propto r^{-2}$ and not with $B \propto r^{-3}$, as for a dipole field. This is of no concern in the present study because we focus essentially on geometrical properties and not on the energetics related to the electromagnetic field strength or dissipation and the associated particle dynamics. The most important feature of the split monopole is the position of its equatorial current sheet. This infinitely thin sheet is actually accurately localized by a simple expression given by a two-dimensional surface expressed in spherical polar coordinates $(r, \vartheta, \varphi)$ as

$r_{\mathrm{s}}(\vartheta, \varphi, t)=\beta_{\mathrm{v}} r_{\mathrm{L}}\left[ \pm \arccos (-\cot \vartheta \cot \alpha)+\frac{c t}{r_{\mathrm{L}}}-\varphi+2 \ell \pi\right]$,

where $\Omega$ is the stellar rotation rate, $c$ the speed of light, $\alpha$ the inclination of the split monopole, $\beta_{\mathrm{v}}=V / c$ the wind speed, $r_{\mathrm{L}}=c / \Omega$ the radius of the light cylinder, $t$ the time as measured by a distant observer at rest, and $\ell$ an integer. The current sheet is connected to the stellar surface by monopolar magnetic field

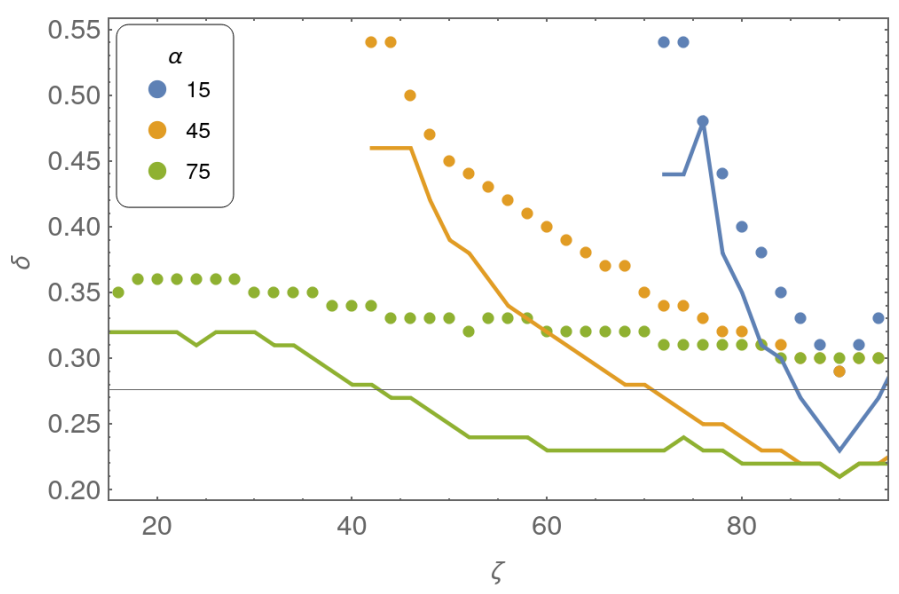

Fig. 1. Time lag $\delta$ between the radio and the closest gamma-ray peak for the split monopole model (dots) and for the dipole model (solid lines) for $\alpha=\left\{15^{\circ}, 45^{\circ}, 75^{\circ}\right\}$.

lines. To a very good approximation we assume that the wind moves radially at exactly the speed of light, $V=c$.

The simultaneous time-aligned radio and gamma-ray pulse profile evolution with the geometric configuration are extensively computed in Pétri (2011). The main features of this emission was a radio time lag $\delta$ connected to the gamma-ray peak separation $\Delta$ (if both gamma peaks are visible) expressed by

$\delta \approx \frac{1-\Delta}{2}$.

Moreover, the gamma-ray peak separation $\Delta$ depends only on $\alpha$ and the inclination of the line of sight $\zeta=\alpha+\beta$. These parameter were found to be related by

$\cos (\pi \Delta)=|\cot \zeta \cot \alpha|$.

These expressions are derived analytically with some approximations detailed in Pétri (2011). According to Eq. (4) the gamma-ray peak separation $\Delta$ is not independent of the radio time lag $\delta$. This formula is actually a simple consequence of the geometrical behaviour and symmetries of the striped wind emission properties related to the polar cap radio emission. It assumes that the radio emission emanates from deep within the light cylinder. In reality, as we show in this paper, we must add an additional delay due to the variable radio emission height from pulsar to pulsar. Equation (5) correlates $\alpha$ and $\zeta$ depending on the peak separation $\Delta$ independently of the radio properties. Here again, this formula is derived from pure geometrical considerations related to the current sheet structure as given by Eq. (3).

In the present work we compute numerically the radio and gamma-ray light curves assuming a Gaussian beam shape around the polar cap for radio emission up to the actual emission height at approximately $0.05 r_{\mathrm{L}}$ and a thin current layer around the current sheet depicted by Eq. (3) for several inclination angles $\alpha$. The results for the radio time lag $\delta$ are shown as coloured dotted points in Fig. 1 for $\alpha=\left\{15^{\circ}, 45^{\circ}, 75^{\circ}\right\}$. The associated gammaray peak separation $\Delta$ is shown as coloured dotted points in Fig. 2, which shows the good agreement between our simulations and the analytical expectation in Eq. (5), also shown as dashed coloured lines. The solid lines correspond to the dipole model, see below.

As a check of the accuracy of relation Eq. (4), we plotted the sum $\delta+\Delta / 2$ in Fig. 3. It always lies around 0.55 , and therefore remains close to the expected value of 0.5 whatever the geometry of the magnetosphere (arbitrary $\zeta$ and $\alpha$ ). 


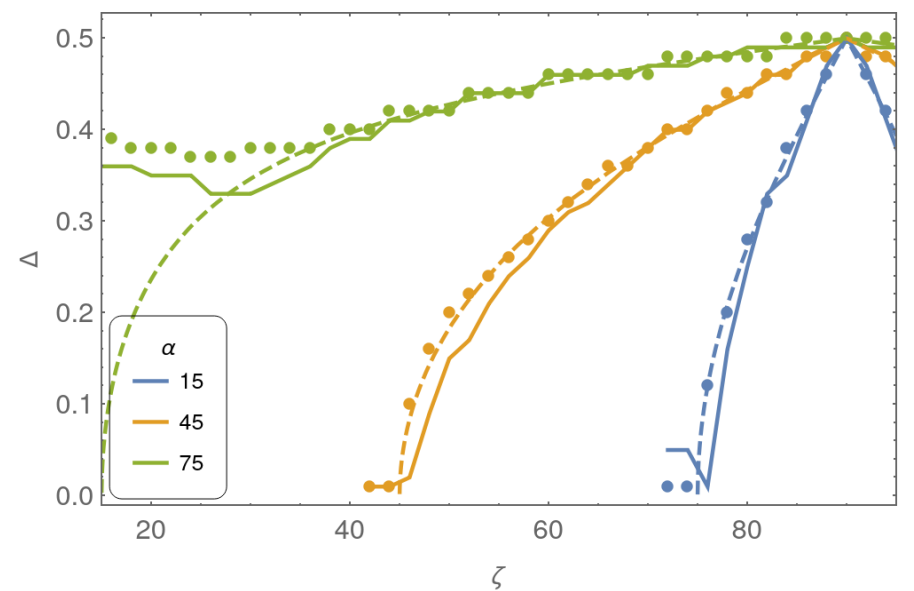

Fig. 2. Gamma-ray peak separation $\Delta$ for the split monopole model (dots) and for the dipole model (solid lines) for $\alpha=\left\{15^{\circ}, 45^{\circ}, 75^{\circ}\right\}$. The dashed lines represent the expectations from Eq. (5).

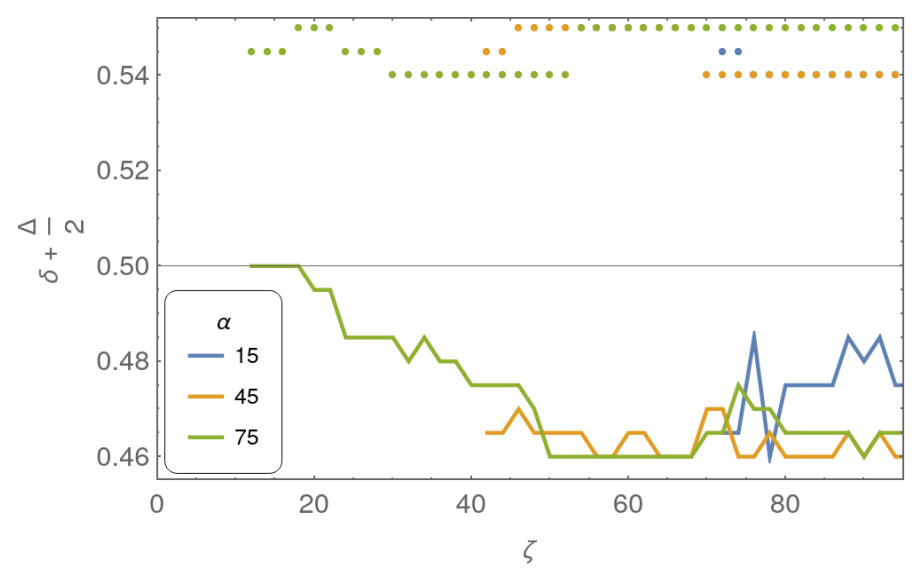

Fig. 3. Sum $\delta+\Delta / 2$ for the split monopole model (dots) and the dipole model (solid lines) for $\alpha=\left\{15^{\circ}, 45^{\circ}, 75^{\circ}\right\}$. The horizontal line shows the expect theoretical value of $1 / 2$.

\subsection{Dipole magnetosphere}

The split monopole gives a good first guess to the structure of the striped wind. However, it does not properly connect the stationary region in the vicinity of the stellar surface to the wave zone outside the light cylinder. A more realistic case must take into account the dipole nature of the field inside the light cylinder and not a split monopole. Therefore, in order to estimate the discrepancy between the split monopole results shown in the previous section and the physical situation of a true magnetosphere, we use the solutions from force-free numerical simulations of pulsar magnetospheres, treating self-consistently the electromagnetic field, and relying on our previous publications in Pétri (2012).

However, we note that young radio pulsars with periods above several tenths of milliseconds are difficult to model numerically because the ratio of the neutron star radius $R$ to the light cylinder radius $r_{\mathrm{L}}$ is very low $a=R / r_{\mathrm{L}} \ll 1$. Because the simulation box must resolve all scales from the neutron star size to the light cylinder length, numerical simulations require very high resolutions in 3D rendering it impossible to reckon the electromagnetic field with a decent computational time. Therefore, in all simulations the ratio $R / r_{\mathrm{L}}$ is artificially increased to millisecond periods in order to get tractable runs. However,
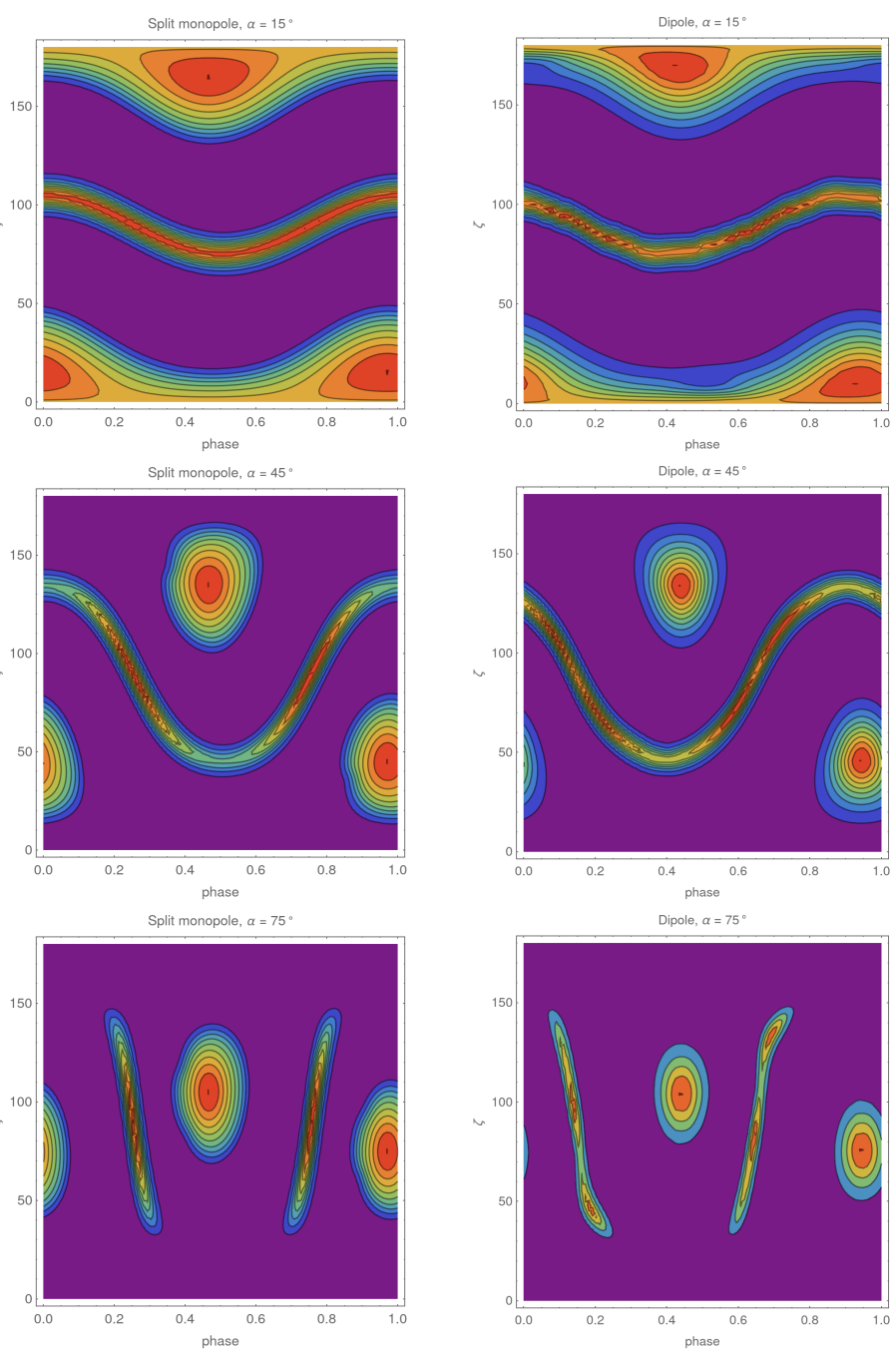

Fig. 4. Gamma-ray sky maps for the split monopole, left column, and the dipole magnetosphere, right column for $\alpha=15^{\circ}, 45^{\circ}, 75^{\circ}$.

such high ratios will not significantly impact the global magnetospheric geometry because variations are expected to scale as $\left(R / r_{\mathrm{L}}\right)^{2}$. For instance, in the Deutsch solution (Deutsch 1955) the spindown correction introduces a factor $\left(1-a^{2}\right)$; meanwhile, the polar cap size decreases as $\sqrt{a}$ without significant changes in their shape (homothetic transformations) (see e.g., Pétri 2018). Therefore, without loss of precision, we can use a ratio $a=0.2$, as was done in our force-free runs to compute young pulsars emission properties to good accuracy. An additional time lag can be added if necessary due to time of flight propagation effects.

We constructed a set of pulsar dipole magnetospheres with $a=0.2$ and obliquities $\alpha$ ranging from $0^{\circ}$ to $90^{\circ}$ in steps of $5^{\circ}$. Then we computed the polar cap shapes, localizing the last open field lines as well as the current sheet outside the light cylinder. The observer line of sight $\zeta$ ranges from $0^{\circ}$ to $180^{\circ}$ in steps of $2^{\circ}$. Some relevant sky maps for split monopole and dipole magnetospheres are shown in Fig. 4.

As done in the previous section for the split monopole, we computed the radio time lag $\delta$, shown as coloured solid lines in Fig. 1. The associated gamma-ray peak separation $\Delta$ is plotted as coloured solid lines in Fig. 2. Here again, we found a good agreement between the dipole model and the analytical expectations in Eqs. (4) and (5). We finally also checked the deviation for the simple law Eq. (4) by computing $\delta+\Delta / 2$ for all 
configurations. Remarkably, we found only a small deviation with a value between 0.46 and 0.5 instead of the theoretical value of 0.5 (see the coloured solid lines in Fig. 3).

Consequently, we have a simple tool to quickly guess the geometry of any radio loud gamma-ray pulsar by measuring its radio lag $\delta$ and gamma-ray peak separation $\Delta$. However, the angles $\zeta$ and $\alpha$ remain degenerate because a continuum of couples $(\zeta, \alpha)$ give the same results. In order to leave the degeneracy, we must scrutinize individually each pulsar by fitting its gammaray light curve. Then, as an a posteriori check, we verify its compatibility with measurements of the radio polarization position angle. This helps to drastically narrow down uncertainties in the geometrical configuration.

The emission height, although situated at about $5 \%$ of $r_{\mathrm{L}}$, is not firmly constrained. There is still a slight freedom to shift the radio time lag to the leading or trailing direction depending on the exact location with respect to our fiducial point. In our simulations we assumed a radio beam radiated in the radial direction at a distance $h_{0}$ from the stellar centre. This height was numerically fixed to $h_{0} / r_{\mathrm{L}}=0.2$ for the dipole simulations. If the radio emission emanates from a distance $h_{1}$ from the stellar centre, the time of flight delay compared to the fiducial altitude $h_{0}$ is

$\Delta t=\frac{h_{0}-h_{1}}{c}$

corresponding to a phase shift in the light curve amounting to

$\phi_{\mathrm{r}}=\frac{h_{0}-h_{1}}{2 \pi r_{\mathrm{L}}}$.

Because the radio pulse profile is taken as phase zero for synchronization purposes, the gamma-ray light curves move in the opposite direction, to earlier phases with respect to the radio pulse profile, and therefore $\phi_{\mathrm{s}}=-\phi_{\mathrm{r}}$. Consequently, allowing emission deeper within the magnetosphere $h_{1}<h_{0}$ shifts the gamma-ray pulse profile to earlier phases with a negative additional delay $\phi_{\mathrm{s}}<0$ compared to our simulated time-aligned gamma-ray profiles. In the opposite case of higher emission altitudes $h_{1}>h_{0}$, the gamma-ray pulse profile shifts to later phases with a positive delay $\phi_{\mathrm{s}}>0$. Therefore, in all of our fits we added an offset phase $\phi_{\mathrm{s}}$ in order to take this uncertainty into account, as well as a possible lack of data in the middle of the radio pulse profile. See also the discussion in Benli et al. (2021). Knowing that emission heights are about $h_{1} \approx 0.05 r_{\mathrm{L}}$, this offset is expected to be around $\phi_{\mathrm{s}} \approx-0.15 / 2 \pi \approx-0.02$. As can be computed from Eq. (7), the phase shift induced by uncertainties in the radio emission height is weak, at most $2 \%$ of the period. Including aberration and/or altitude dependent magnetic field sweep back (Phillips 1992) will at most double or triple this value. The good news is that we do not need an accurate location of the radio emission site. The bad news is that larger shifts, as we found in our fittings, requires other ingredients to justify the $10 \%$ or $15 \%$ shift in the period. One possibility is to move the emission from the striped wind to larger distances, not starting right at the light cylinder but at two or three times $r_{\mathrm{L}}$. Shifting from $1 r_{\mathrm{L}}$ to $2 r_{\mathrm{L}}$ introduces a time lag (actually an advance in time corresponding to a shift to earlier phases) of approximately $1 / 2 \pi \approx 0.16=16 \%$ of the period.

In order to summarize all possible gamma-ray light curves, single peaked or double peaked, an atlas is shown in Fig. 5 with the full range of obliquities $\alpha$ and line of sight $\zeta$. We note that all intensities are normalized to unity, but in reality we expect much fainter radiation when the observer line of sight does not cross or only grazes the current sheet in the wind. Due to the symmetry of

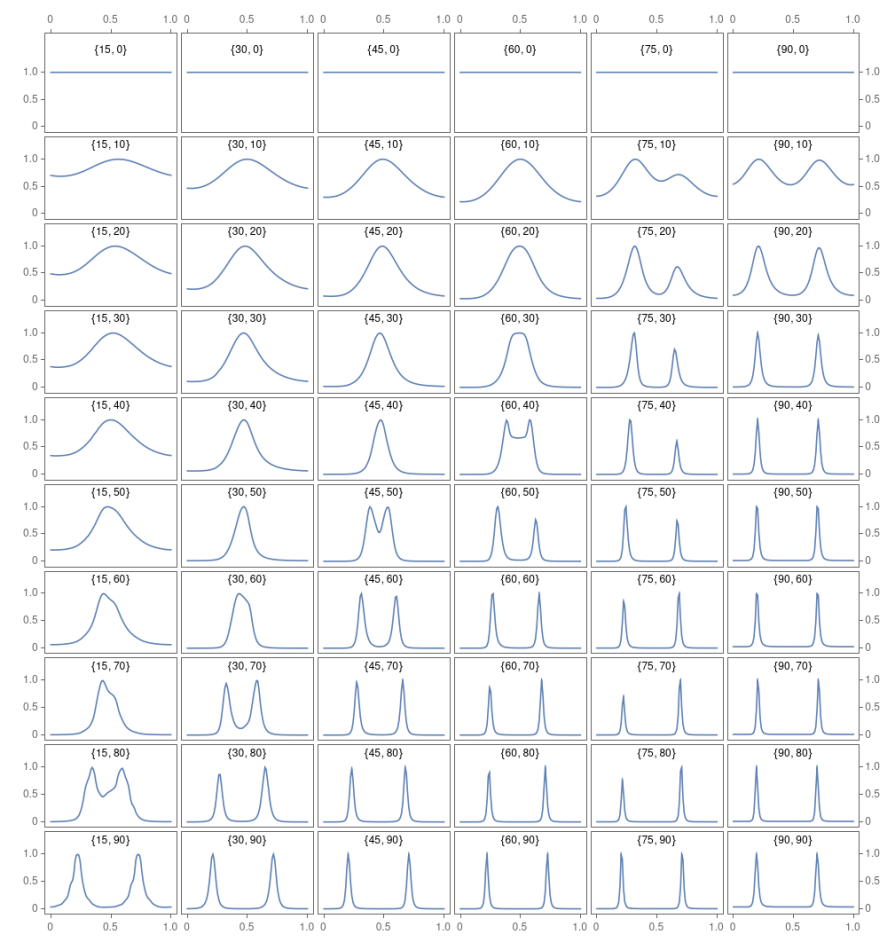

Fig. 5. Atlas of gamma-ray light curves for $\alpha=$ $\left\{15^{\circ}, 30^{\circ}, 45^{\circ}, 60^{\circ}, 75^{\circ}, 90^{\circ}\right\}$ from left to right, and $\zeta=\left\{0^{\circ}, \ldots, 90^{\circ}\right\}$ from top to bottom with steps of $10^{\circ}$ in the format $\{\alpha, \zeta\}$.

the dipole, we do not show the southern hemisphere light curve atlas with either $\alpha>90^{\circ}$ or $\zeta>90^{\circ}$. The radio and gammaray sky maps highlight a north-south symmetry meaning that the configuration $(\alpha, \zeta)$ produces exactly the same light curves as the symmetrical configuration $(\pi-\alpha, \pi-\zeta)$. Another important symmetry connects $(\alpha, \zeta)$ to $(\alpha, \pi-\zeta)$, the latter showing a light curve identical to the former except for a shift in phase of half a period. Therefore, from the knowledge of the sky maps for only the obliquity range $(\alpha, \zeta) \in\left[0^{\circ}, 90^{\circ}\right]^{2}$, we are able to produce any light curve whatever $(\alpha, \zeta) \in\left[0^{\circ}, 180^{\circ}\right]^{2}$. We note that this symmetry is broken when fitting the radio PPA. Very highquality polarization data are able to lift the degeneracy from the gamma-ray sky maps, pinning down the angles to small uncertainties. These conclusions are primordial when studying pulsars for which we expect $\alpha>90^{\circ}$ such as J0742-2822, J0908-4913, and $\mathrm{J} 1702-4128$. However, we use $\alpha \leq 90^{\circ}$ to adjust gamma-ray light curves even if $\alpha$ is constrained to be larger than $90^{\circ}$ from RVM thanks to this symmetry.

Several kinds of profiles are produced. Asymmetric single peaks are obtained for weak inclination angles $\alpha$ and $\zeta$, in the upper left part of the atlas. When moving downwards to the right an unresolved double peak structure appears with two overlapping peaks showing a kind of bridge emission. For the largest angles $\alpha$ and $\zeta$, in the lower right part of the atlas, the two peaks are well separated. We also note that the dominant peak is either the first or the second, depending on the observer line of sight. For instance, the case $\alpha=75^{\circ}$, fifth column, starts with a dominant first peak becoming weaker when the observer looks through the equator, for $\zeta>50^{\circ}$.

The simultaneous observation of radio and gamma-ray pulses is conditioned to the line of sight crossing the radio emission cone. Assuming the formula for a static dipole and setting the emission height at a distance $r$ from the stellar centre, the 


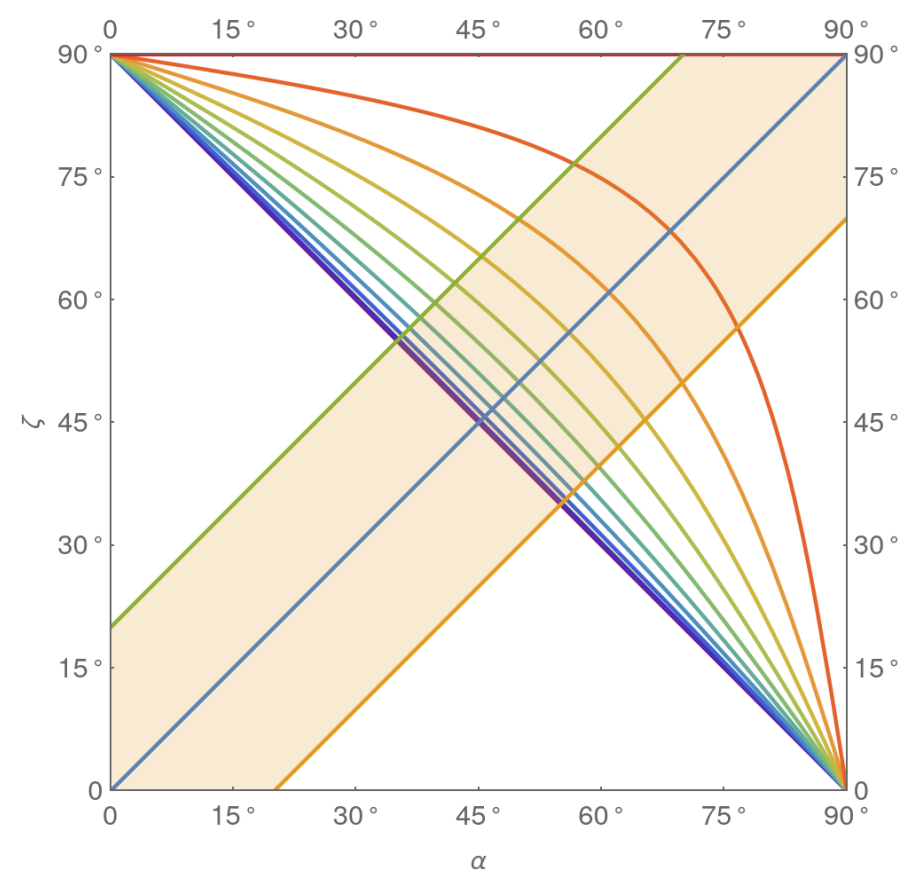

Fig. 6. Isocurves of constant gamma-ray peak separation $\Delta$ depending on $\alpha$ and $\zeta$. The orange shaded area corresponds to observable radio emission with beam half opening angle $\theta_{\mathrm{em}}=20^{\circ}$.

half opening angle of this cone is

$\theta_{\mathrm{em}}=\frac{3}{2} \theta_{\mathrm{pc}} \approx \frac{3}{2} \sqrt{\frac{R}{r_{\mathrm{L}}}} \approx 1.3^{\circ}\left(\frac{P}{1 s}\right)^{-1 / 2}$.

Actually, the radio emission does not escape from the polar caps for young pulsars, but at a substantial height above the stellar surface, around $r \approx 0.05 r_{\mathrm{L}}$ (Mitra 2017). The half opening angle is therefore insensitive to the period and equals

$\theta_{\mathrm{em}}=\frac{3}{2} \sqrt{\frac{r}{r_{\mathrm{L}}}} \approx 20^{\circ}$

This means that the line of sight must not deviate more than $\theta_{\mathrm{em}}$ from the magnetic moment axis $\zeta \in\left[\alpha-\theta_{\mathrm{em}}, \alpha+\theta_{\mathrm{em}}\right]$ or for the angle $\beta \in\left[-\theta_{\mathrm{em}},+\theta_{\mathrm{em}}\right]$. Meanwhile, for gamma rays to be visible, we impose $90^{\circ}-\alpha \lesssim \zeta \lesssim 90^{\circ}+\alpha$. A summary of relevant angles in the $(\alpha, \zeta)$ plane is shown in Fig. 6, linking the variation in $\zeta$ to the variation in $\alpha$ for a fixed gamma-ray peak separation $\Delta$. The orange shaded area delimits the region where radio pulse profiles are detected according to the cone opening angle $\theta_{\mathrm{em}}$. Radio-loud gamma-ray pulsars are located in the upper right part of this shaded area, for angles $\alpha \gtrsim 45^{\circ}$. Actually, for each pulsar with known $\Delta$, we can constrain the obliquity $\alpha$ by setting an interval $\left[\alpha_{\min }, \alpha_{\max }\right]$, as shown in Fig. 7. Higher peak separations imply higher obliquities, tending towards $90^{\circ}$. The blue points correspond to the results of the fits performed in Sect. 4.

Radio-loud single gamma-ray peak pulsars are seen when the observer line of sight grazes the edges of the current sheet within the striped wind. This occurs whenever $\alpha+\zeta \approx 90^{\circ}$. Moreover, this line of sight must cross the radio beam, and therefore $|\zeta-\alpha| \lesssim$ $\theta_{\mathrm{em}}$. This puts severe constraints on $\alpha$, namely $\left|\alpha-45^{\circ}\right| \lesssim \theta_{\mathrm{em}} / 2$. In our case with $\theta_{\mathrm{em}}=20^{\circ}$ we get $(\alpha, \zeta) \in\left[35^{\circ}, 55^{\circ}\right]^{2}$, which corresponds to the area around $\Delta=0$ in Fig. 7 .

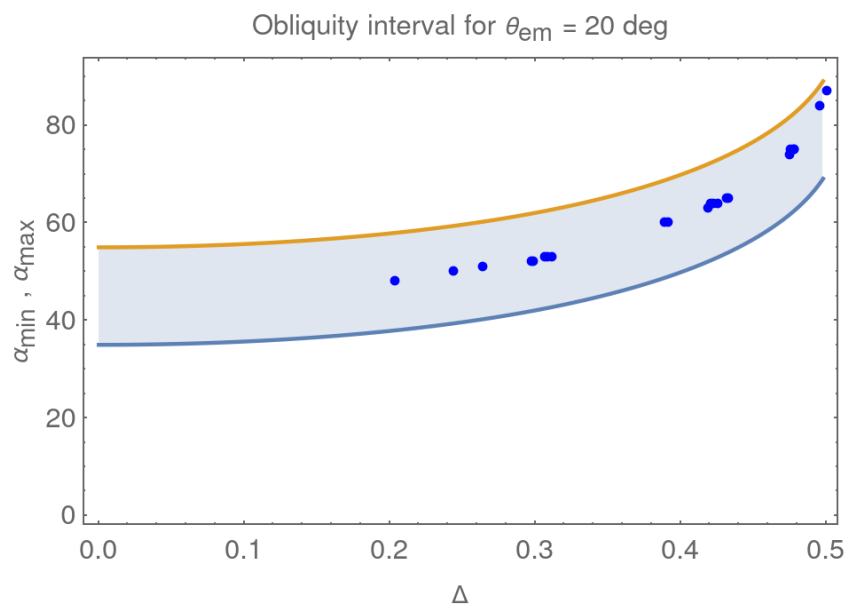

Fig. 7. Constraint on the obliquity for a radio-loud gamma-ray pulsar with two peaks separated by $\Delta$. The blue points correspond to the results of the fits performed on the pulsar sample chosen in this work (see Sect. 4).

\section{Results}

In this section we describe our fitting method, the young pulsar population used in our study and eventually discuss the results of the best geometry within the combined gamma-ray striped wind and radio rotating vector model. Implications for the emission sites are also discussed.

To keep the gamma-ray emission model as simple as possible, we use exactly the same size for the current sheet emission for all pulsars, integrating photon emissivity in a spherical shell comprised between the radius $r=r_{\mathrm{L}}$ and $r=3 r_{\mathrm{L}}$. We recall moreover that these gamma rays are emitted tangentially to the current sheet in its rest frame, but due to Lorentz boosting to the observer frame, this radiation is directed almost radially for that observer.

\subsection{Fitting method}

Our fitting method closely follows the technique used by Benli et al. (2021). The important features to be matched are the radio-gamma-ray time lag and the gamma-ray peak separation (if both peaks are visible) and the gamma-ray light curve profiles. The precise radio pulse profile is irrelevant to our study because we do not investigate in depth the radio emission mechanism. We only require an estimate of its emission altitude and assume a Gaussian shape to accurately localize the radio peak phase taken by definition as phase zero. Most importantly, we fit as properly as possible the time-aligned gamma-ray light curves in accordance with the radio peak synchronization. According to the pulsar gamma-ray catalogue (Abdo et al. 2013), the synchronization performed by the Fermi/LAT collaboration varies from pulsar to pulsar for several reasons, mainly because the determination of the centre of the radio pulse profile is problematic. In our investigations, we do not suffer from such indeterminacy because we take the plane formed by the magnetic axis and the rotation axis as a fiducial plane which has phase zero by convention. In this way we get a homogeneous fitting procedure for all pulsars in our sample. The $\chi^{2}$ introduced for the gamma-ray light curve fitting is expressed as

$\chi^{2}=\sum_{i} \frac{\left(I_{\mathrm{i}}^{\mathrm{obs}}-I_{\mathrm{i}}^{\text {model }}\right)^{2}}{\sigma_{\mathrm{i}}^{2}}$, 
where $I_{i}^{\text {obs }}$ is the observed gamma-ray intensity, $\sigma_{i}^{2}$ its associated error for the $i$ th phase bin, and $I_{i}^{\text {model }}$ the model intensity at the same observational bin. As the observational phase bins do not coincide with the theoretical phase bins, we interpolate the theoretical light curves at the observational phase bins.

\subsection{Pulsar sample}

Our sample of young and radio-loud gamma-ray pulsars is guided by the existence of good quality gamma-ray light curves and if possible in conjunction with good radio polarization data in order to fit the polarization position angle (PPA) with the rotating vector model. Our choice implies selecting pulsars with periods above approximately $30 \mathrm{~ms}$ in order to ensure radio photon production at high altitude above the polar cap where the dipole magnetic field approximation holds accurately. The A-R effect measured in those pulsars indeed constrains the emission height to a fraction of the light cylinder radius. Bearing in mind all these constraints, we arrive at a reasonable sample of 31 pulsars summarized in Table 1. The pulsar period ranges from $39 \mathrm{~ms}$ to more than $400 \mathrm{~ms}$. Except for seven pulsars, they all show a double gamma-ray pulse profile with $\Delta$ in the range $0.2-0.5$. The gamma-ray peak time lag goes from 0.06 to 0.63 .

\subsection{Joined RVM and gamma-ray fits}

We start with the sub-sample of pulsars having a reasonable RVM fit to constrain the two angles $\alpha$ and $\beta=\zeta-\alpha$. The gammaray light curves are extracted from the second pulsar catalogue (Abdo et al. 2013).

For all these pulsars, we show in the same figure first the radio pulse profile with the best RVM fit, then the radio and gamma-ray $\chi^{2}$ fit, and finally the best radio and gamma-ray light curves predictions compared to observations. We briefly go through all these pulsars below.

PSR J0631+1036. With a period of $288 \mathrm{~ms}$ this pulsar shows something that looks like one gamma-ray peak or an unresolved double peak. Its radio pulse profile and the corresponding PPA are shown in the top panel of Fig. 8. The $\log \chi^{2}$ contour plots for radio polarization fits is shown in coloured contours and the gamma-ray light curve fits in solid lines in the middle panel of Fig. 8. The red cross indicates the obliquity and the inclination angle for the best joined fit. The corresponding radio and gamma-ray light curves are overlapped with observations in the bottom panel of Fig. 8. The gamma-ray best fit light curve resembles an unresolved double peaked profile. However, a better signal-to-noise ratio is required to firmly distinguish between a single- and a double-peak structure. Nevertheless, the joined radio and gamma-ray fit severely constrains the geometry of $\mathrm{J} 0631+1036$ because the most likely regions in the $(\alpha, \beta)$ plane are very different for the two wavelengths. The gamma-ray fit is good and consistent with radio polarization data. A small offset is required $\phi_{\mathrm{s}}=0.01$ for $\alpha=40^{\circ}$ and $\zeta=36^{\circ}$. We note that other fits are not excluded because the best radio and best gamma-ray fits are not always strictly compatible. Therefore, depending on the weight of each wavelength for defining a global $\chi^{2}$ fit, we arrive at slightly different geometries. For conciseness, we do not plot them.

$P S R$ J0659+1414. Figure 9 shows this clear single gammaray peaked pulsar with period $385 \mathrm{~ms}$. Good radio polarization data in the top panel of Fig. 9 constrain the angles through the $\chi^{2}$ contour plots of radio and gamma-ray observations, as for J0631+1036 (middle panel of Fig. 9). The gamma-ray pulse pro-
J0631+1036, $1400 \mathrm{MHz}$


Fig. 8. Combined radio polarization and gamma ray fitting of J0631+1036. Top: radio polarization data with the best RVM fit of J0631+1036. Middle: $\log \chi^{2}$ contour plots, in coloured contours for radio polarization fits, and in solid coloured lines for gamma-ray light curves. The red cross indicates the best joined radio-gamma-ray fit. Bottom: associated gamma-ray light curve for the geometry is given by the red cross.

file looks very symmetric and is well reproduced by our model, showing a symmetrical shape with respect to leading and trailing wings. The best fit shown by the red cross in the middle panel 

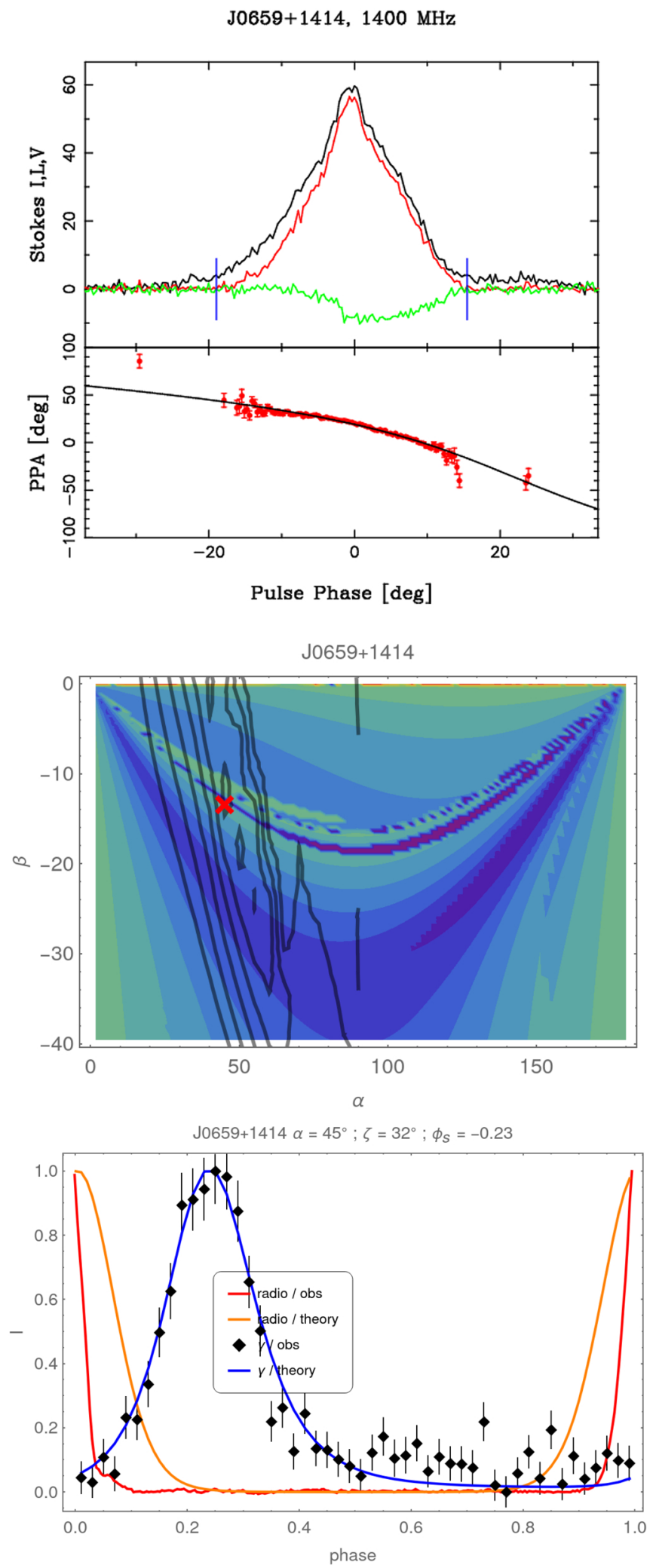

Fig. 9. Same as Fig. 8, but for J0659+1414. The gamma-ray best fit coincides with the radio polarization best fit.

coincides with the radio constraint. However, the additional offset of $\phi_{\mathrm{s}}=-0.23$ is large with a geometry given by $\alpha=45^{\circ}$ and $\zeta=32^{\circ}$.

PSR J0742-2822. This pulsar has mainly one gamma-ray pulse with the largest radio time lag of 0.627 and a period of
$167 \mathrm{~ms}$. The radio polarization swing is clearly visible in the top panel of Fig. 10. The contour plots of $\log \chi^{2}$ in radio and gamma rays overlap in a small region, as seen in the second panel from the top. It favours an obliquity $\alpha$ larger than $90^{\circ}$. Strictly speaking, we did not perform simulations for $\alpha>90^{\circ}$, but we can use the symmetry of the gamma-ray light curves to find the high-energy profiles for $\alpha>90^{\circ}$. Our striped wind model is symmetric about the equatorial plane, meaning that the configuration $(\alpha, \zeta)$ gives exactly the same light curves as the configuration $(\pi-\alpha, \pi-\zeta)$. In other words, the radio fit $(\alpha, \beta)$ gives the same results as the fits for $(\pi-\alpha,-\beta)$. Therefore, for the gammaray light curve, we use a kind of reciprocal to the $\chi^{2}$ obtained from the original radio data by changing $\alpha$ to $\pi-\alpha$ and $\beta$ to $-\beta$. Doing this we get the middle panel of Fig. 10 showing the best gamma-ray fit coincident with radio polarization. It corresponds to $\alpha=40^{\circ}$ and $\beta=4^{\circ}$. Reversing the symmetry argument, the real best fit is given by an offset equal to $\phi_{\mathrm{s}}=0.16$ for $\alpha=140^{\circ}$ $\left(180^{\circ}-40^{\circ}\right)$ and $\zeta=136^{\circ}\left(\beta=-4^{\circ}\right)$.

PSR J0835-451. The Vela pulsar with period $89 \mathrm{~ms}$ shows two prominent and well-defined gamma-ray peaks surrounding a weaker third peak wandering in phase with energy (bottom panel of Fig. 11). Our model can only produce two peaks, so we discard the third peak. The radio polarization can be reasonably fitted with the RVM model, but only around the steepest gradient, top panel of Fig. 11. The middle panel shows the $\log \chi^{2}$ contour plots for radio polarization and gamma-ray light curves with the red cross lying slightly apart from the RVM constraint. The two prominent gamma-ray peaks are well fitted with the geometry shown in the bottom panel. The offset is $\phi_{\mathrm{s}}=-0.1$ for $\alpha=65^{\circ}$ and $\zeta=58^{\circ}$.

PSR J0908-4913. This pulsar of $107 \mathrm{~ms}$ is another example of a double-peaked gamma-ray pulsar, although noisy (see bottom panel of Fig. 12). It also shows a less prominent interpulse in radio at phase 0.5 , suggesting that it is close to an orthogonal rotator (top panel). The RVM constraints are shown in the middle panel of Fig. 12, clearly highlighting the orthogonal nature of the pulsar with a line of sight passing close to the magnetic axis because $-3^{\circ}<\beta<-5^{\circ}$ (see also Kramer \& Johnston 2008 for similar conclusions). As for J0742-2822, the obliquity $\alpha$ is larger than $90^{\circ}$. We use again the symmetry argument to find the best gamma-ray fit with $\alpha^{\prime}=85^{\circ}$ and $\zeta^{\prime}=88^{\circ}$. The two peak maximum intensity values are different and not fully reproduced by our model. Nevertheless, the two radio peaks are visible for an offset of $\phi_{\mathrm{s}}=-0.08$ and the real angles are $\alpha=95^{\circ}$ and $\zeta=92^{\circ}$, depicted by the red cross on the RVM fit contour, thus it is indeed an orthogonal rotator. Our predicted radio interpulse intensity is much higher than the observer flux. A proper understanding of this effect, if not geometric, requires knowledge of the radio emission mechanism which is beyond the scope of this paper.

PSR J1048-5832. This is another bright gamma-ray pulsar of period $124 \mathrm{~ms}$, possessing very good radio polarization data (top panel of Fig. 13), leading to an accurate $\chi^{2}$ plot, as seen in the middle panel of Fig. 13. It shows two narrow and prominent gamma-ray pulses well fitted by the red cross area coincident with radio polarization constraints. The overlapping region therefore severely pins down the geometry of J1048-5832 to be around $\alpha=60^{\circ}$ and $\zeta=68^{\circ}$ with an offset of $\phi_{\mathrm{s}}=-0.12$.

PSR J1057-5226. A single gamma-ray peak with a kind of large plateau or an unresolved double gamma-ray peak is visible for this pulsar (Fig. 14). A radio pulse and an interpulse are seen, possibly making it an almost orthogonal rotator. However, 
J0742-2822, $1400 \mathrm{MHz}$

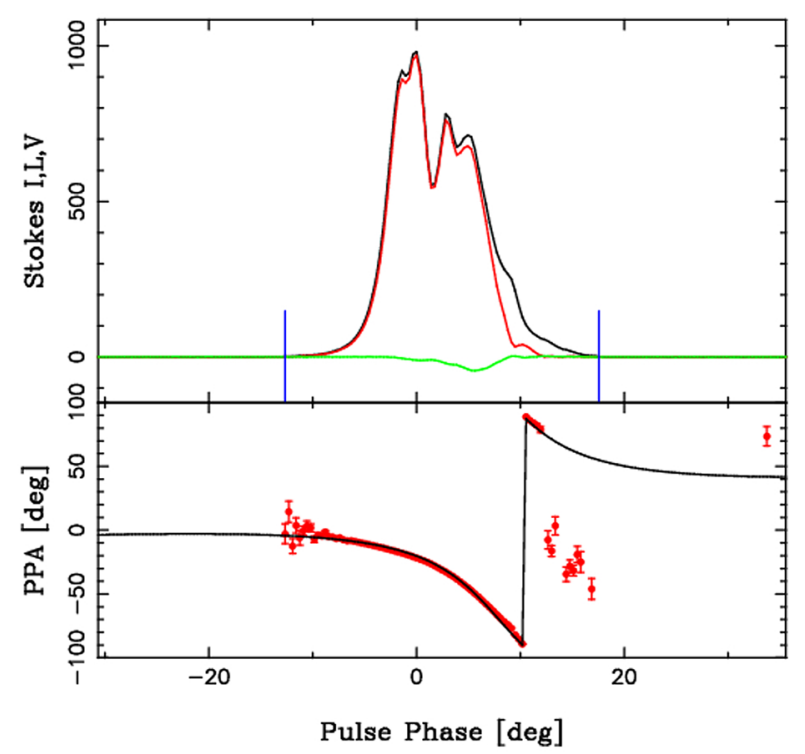

J0742-2822
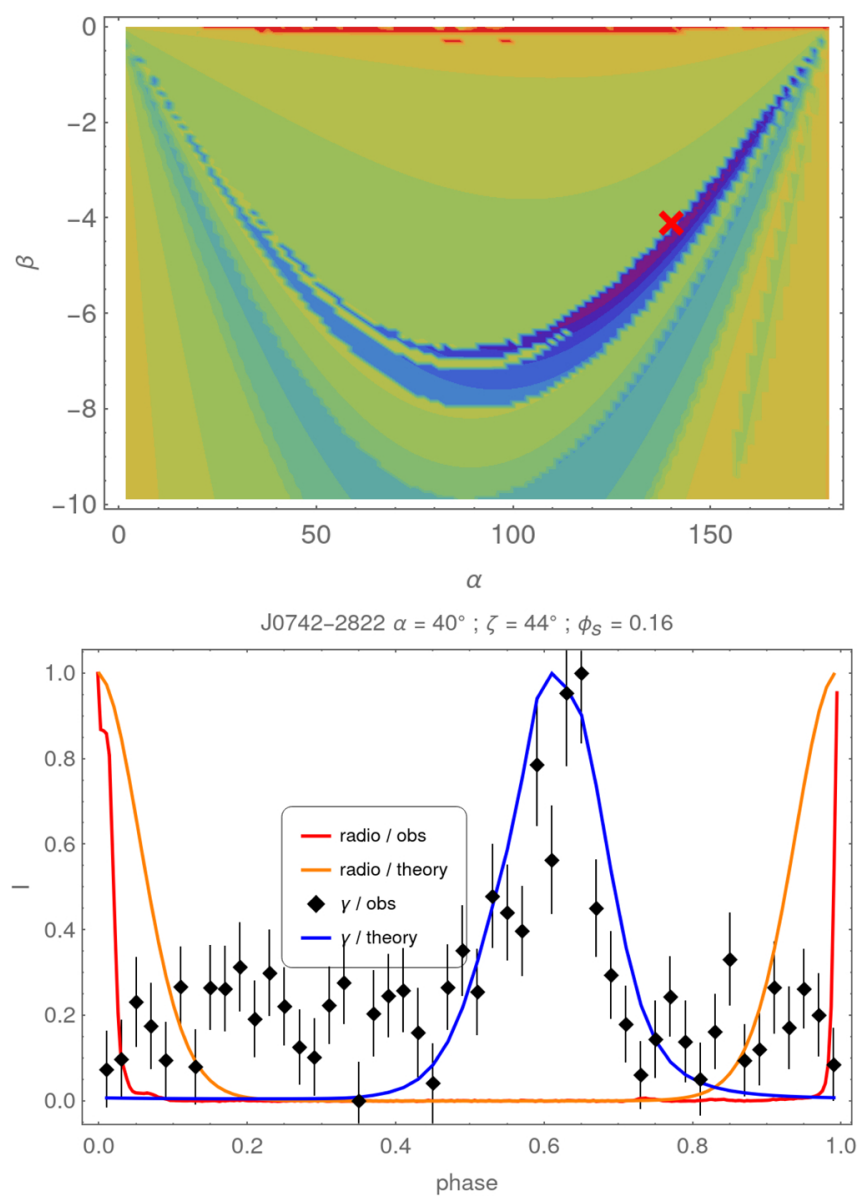

Fig. 10. Same as Fig. 8, but for J0742-2822.

we found a relatively low obliquity of only $\alpha \approx 25^{\circ}$ with $\zeta=44^{\circ}$ and $\phi_{\mathrm{s}}=-0.01$. No radio interpulse is predicted by this geometry. This pulsar does not easily accommodate with our picture of a combined polar cap striped wind emission model. The $\mathrm{RVM}$ fit to the radio polarization, however, is consistent with $\alpha \approx 75^{\circ}$ and $\zeta \approx 110^{\circ}$, which is also the result obtained by
J0835-4510, $1400 \mathrm{MHz}$
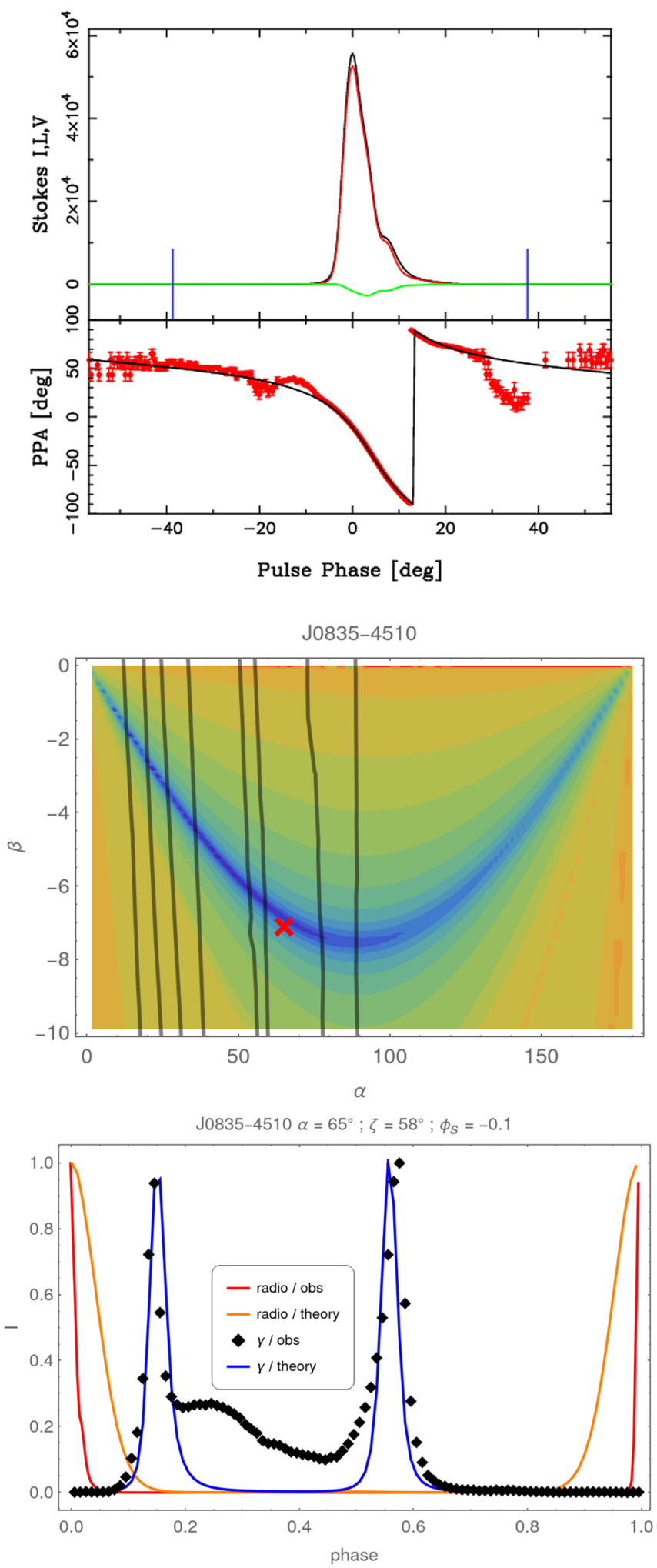

Fig. 11. Same as Fig. 8, but for J0835-451. The third peak is not taken into account.

Weltevrede \& Wright (2009) and is shown in the top panel of Fig. 14. This is the only example of our sample that does not fit into the joint radio and gamma-ray fitting procedure.

PSR J1119-6127. This pulsar with a period of $408 \mathrm{~ms}$ shows a weakly double-peaked gamma-ray profile. Its radio 
J0908-4913, $1400 \mathrm{MHz}$

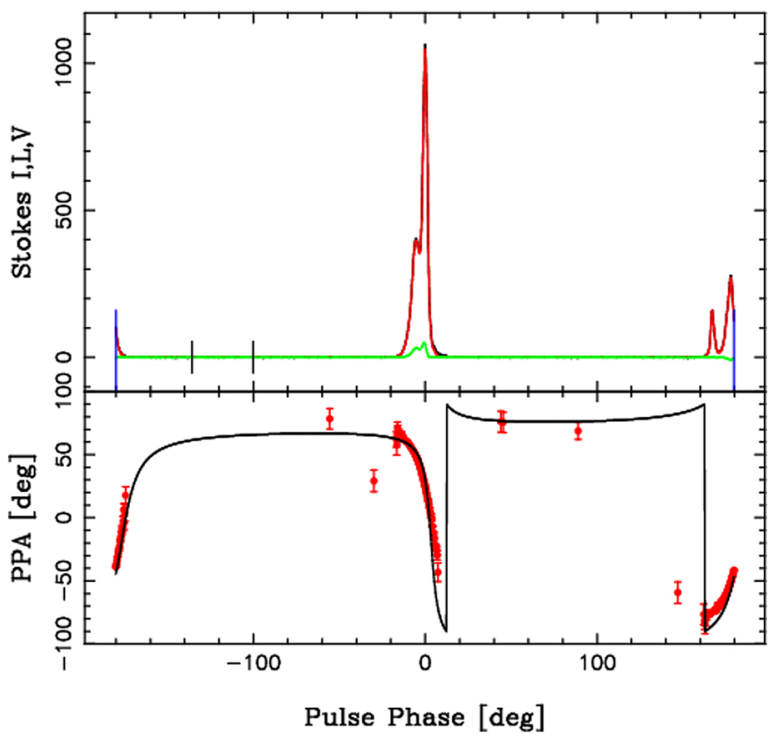

J0908-4913

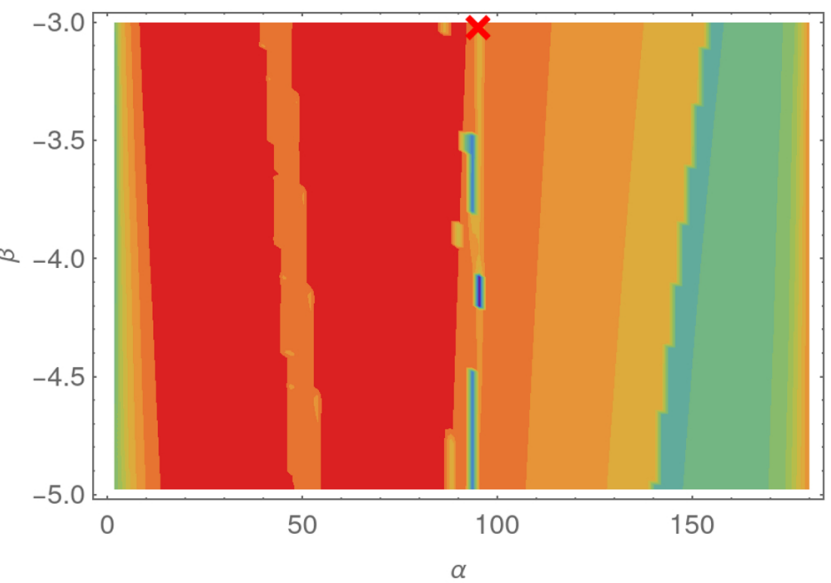

J0908-4913 $\alpha=95^{\circ} ; \zeta=92^{\circ} ; \phi_{S}=-0.08$

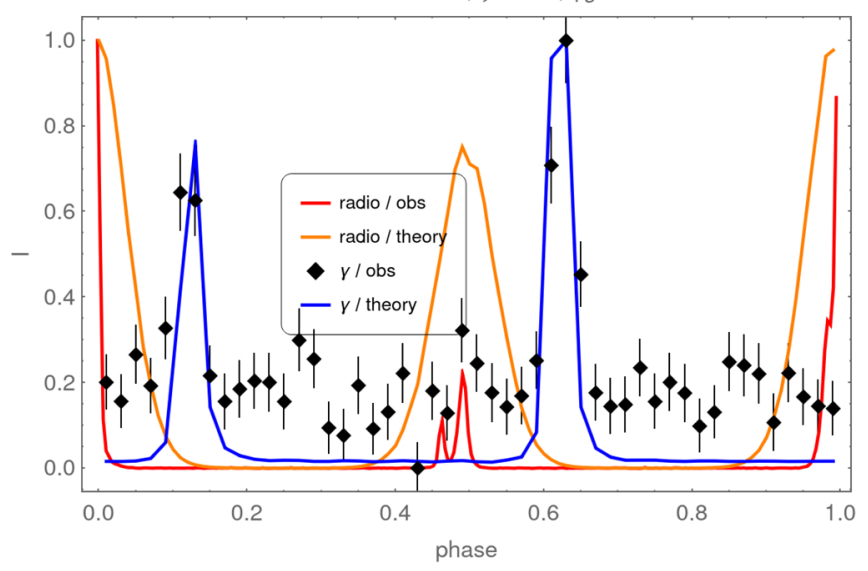

Fig. 12. Same as Fig. 8, but for J0908-4913.

polarization data are noisy (top panel of Fig. 15), implying a large area for the PPA constraint (middle panel). Two distinct joined radio gamma-ray best fits are also possible. One fit leads to a single gamma-ray profile, not shown, and one to an unresolved double gamma-ray light curve (bottom panel, and red cross in middle panel). We had to add an additional phase shift
$\mathrm{J} 1048-5832,1400 \mathrm{MHz}$

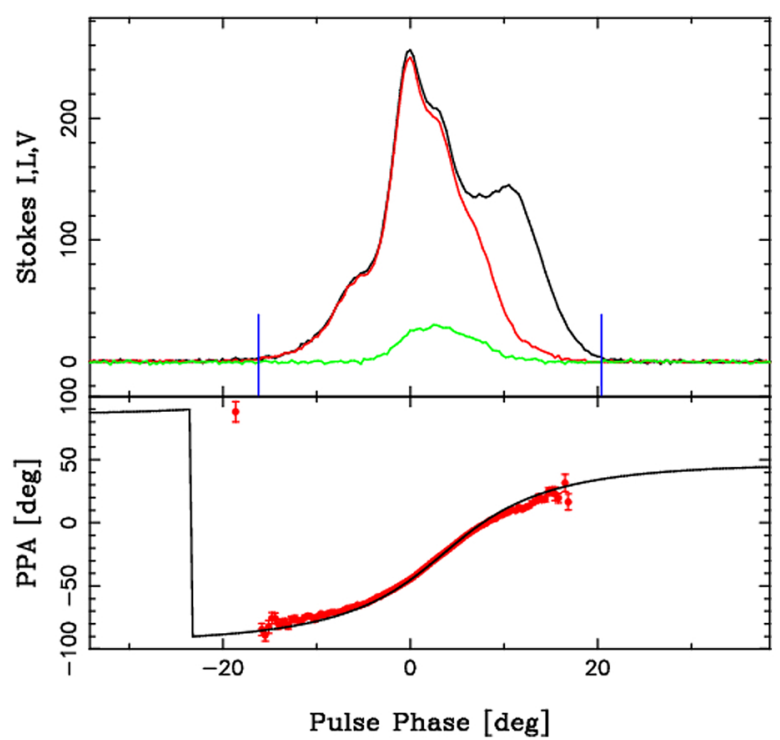

J1048-5832

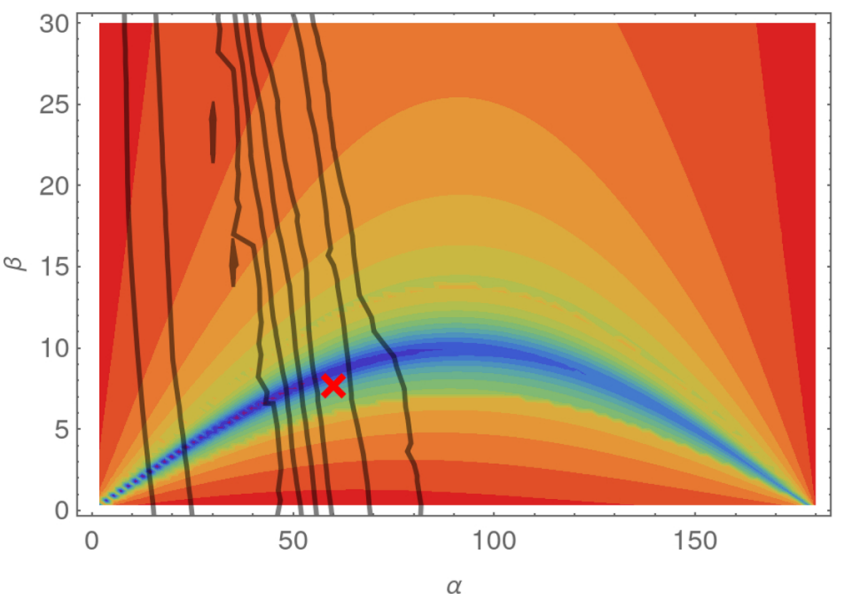

$\mathrm{J} 1048-5832 \alpha=60^{\circ} ; \zeta=68^{\circ} ; \phi_{S}=-0.12$

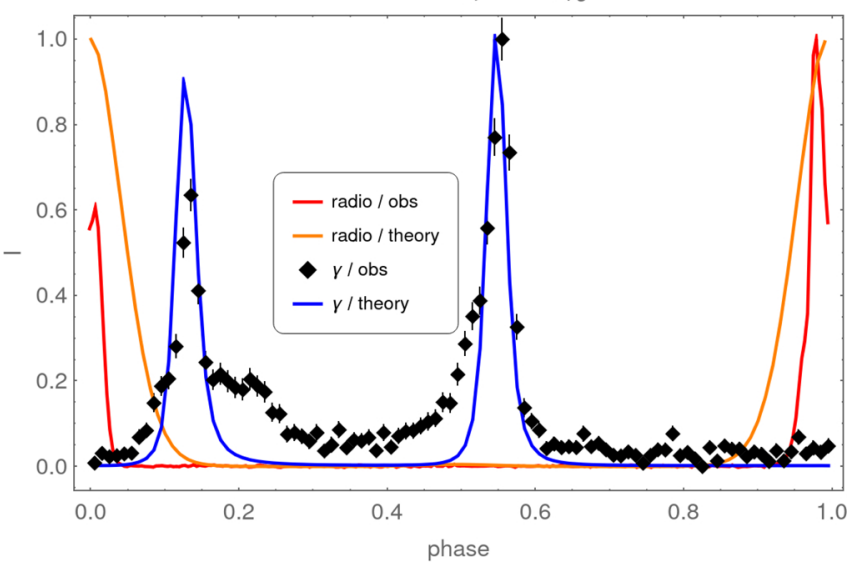

Fig. 13. Same as Fig. 8, but for J1048-5832.

of about $\phi_{\mathrm{s}}=-0.06$ for $\alpha=60^{\circ}$ and $\zeta=40^{\circ}$. Better quality gamma-ray data will certainly favour this second option.

PSR J1357-6429. Similar to the previous pulsar, PSR J1357-6429 is noisy in radio, top panel of Fig. 16 with large uncertainties in the RVM constrain, middle panel. 
J1057-5226, $1400 \mathrm{MHz}$
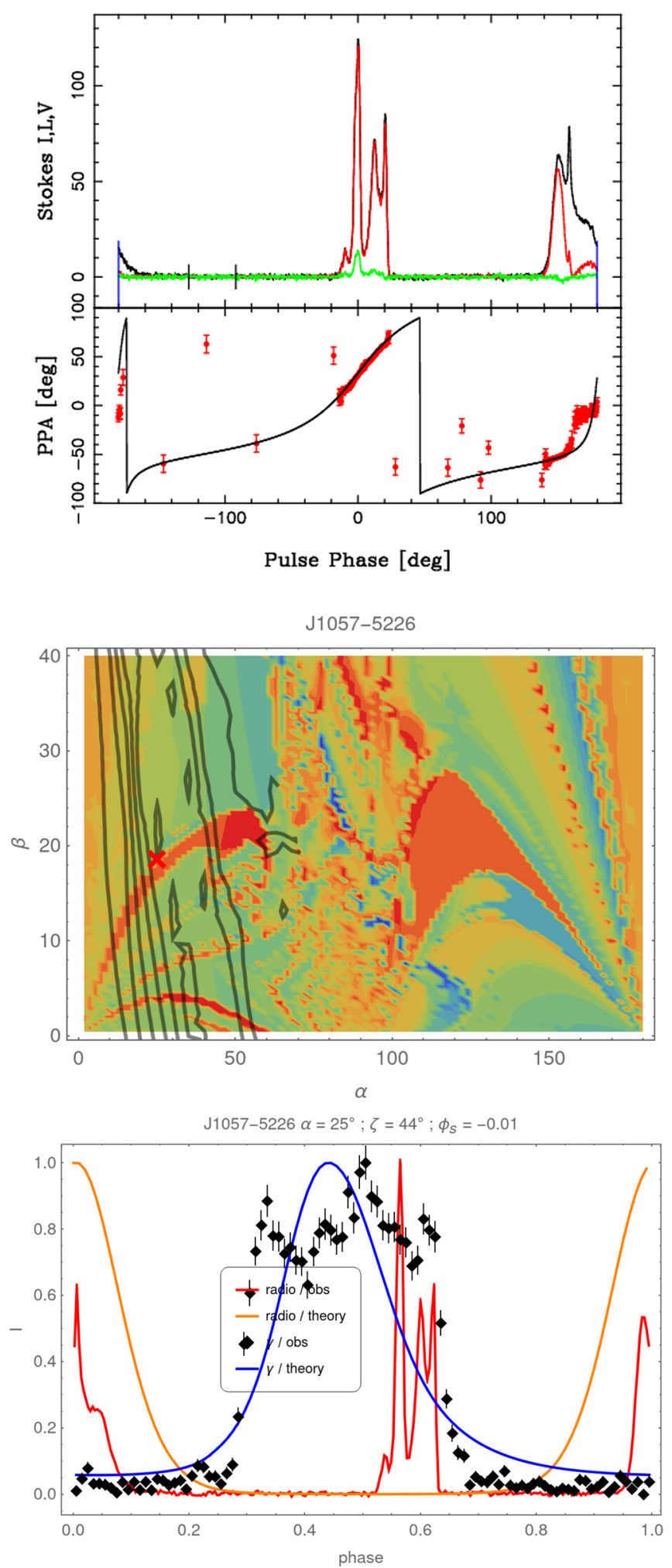

Fig. 14. Same as Fig. 8, but for J1057-5226.

Two options are given by either an unresolved double gammaray peak (not shown) or a single gamma-ray peak (bottom panel). It is another example of a single gamma-ray peak pulsar fitted with a small obliquity. We added an additional phase shift of $\phi_{\mathrm{s}}=-0.09$ for $\alpha=20^{\circ}$ and $\zeta=34^{\circ}$ which also seems the most likely.
$\mathrm{J} 1119-6127,1400 \mathrm{MHz}$



J1119-6127

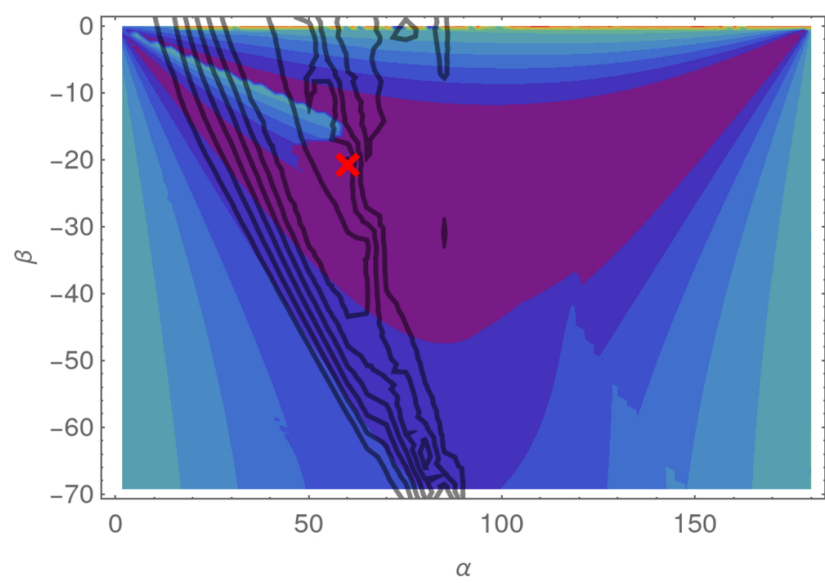

$\mathrm{J} 1119-6127 \alpha=60^{\circ} ; \zeta=40^{\circ} ; \phi_{S}=-0.06$

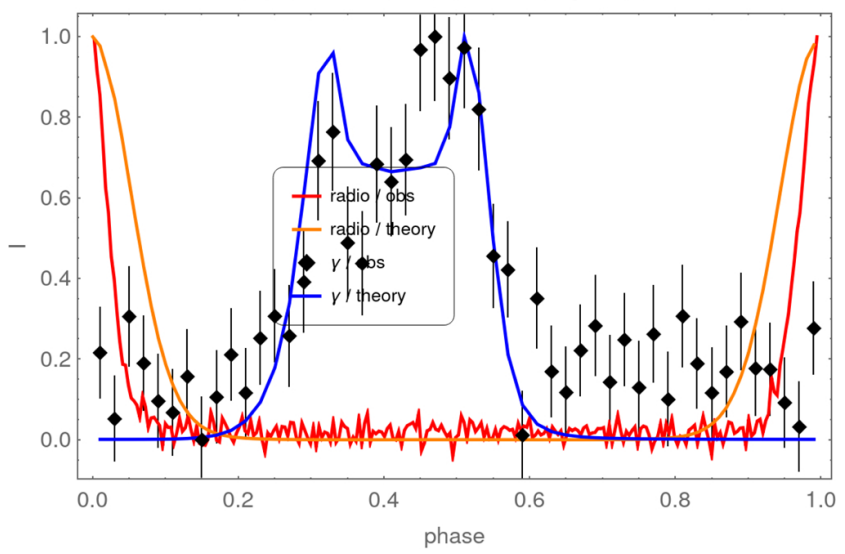

Fig. 15. Same as Fig. 8, but for J1119-6127.

PSR J1420-6048. This double-peaked gamma-ray pulsar possesses an unresolved double gamma-ray peak profile with an asymmetry in the peak intensities (bottom panel of Fig. 17). The good radio polarization data (top panel) furnishes good RVM constraints (middle panel). Most likely it is the geometry given by the red cross that leads to the double-peak profile visible in 
$\mathrm{J} 1357-6429,1400 \mathrm{MHz}$
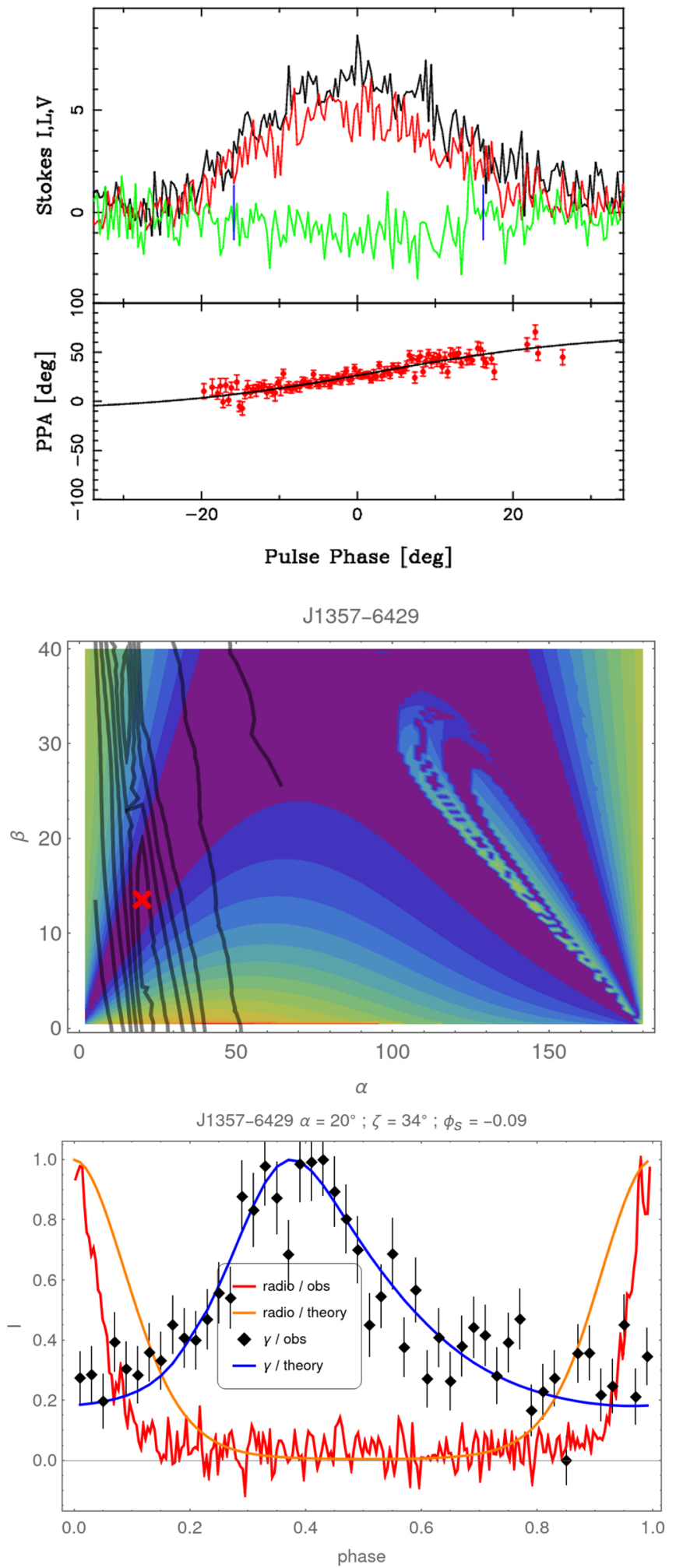

Fig. 16. Same as Fig. 8, but for J1357-6429.

the bottom panel. The best fit configuration has a phase shift of $\phi_{\mathrm{s}}=-0.08$ for $\alpha=45^{\circ}$ and $\zeta=56^{\circ}$.

PSR J1648-4611. The situation for this pulsar is more clear cut. Although the radio polarization data are noisy (top panel of Fig. 18), the joined radio gamma-ray fit leads to a well-defined
$\mathrm{J} 1420-6048,1400 \mathrm{MHz}$

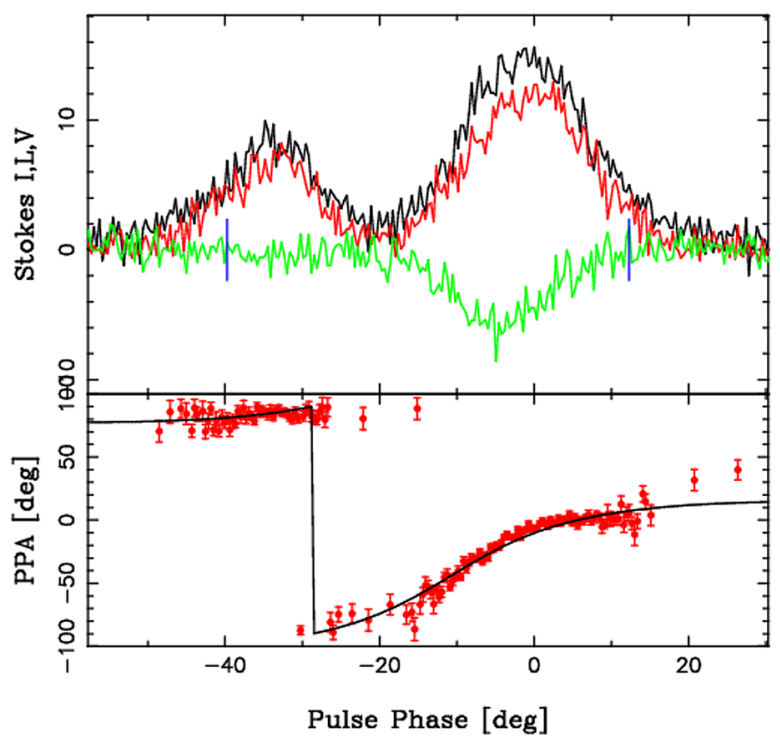

J1420-6048
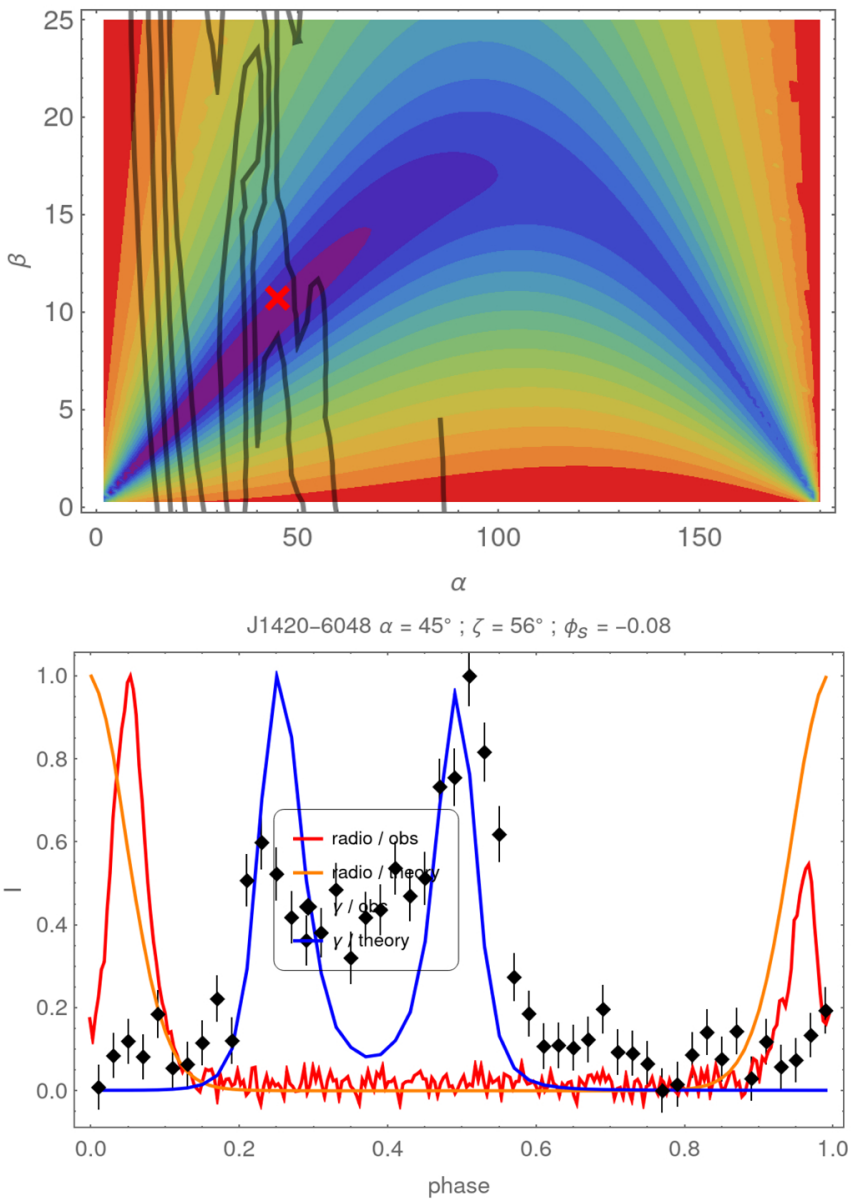

Fig. 17. Same as Fig. 8, but for J1420-6048.

geometry (middle panel). The gamma-ray pulse profile resembles a double peaked curve with a plateau (bottom panel). The corresponding phase shift is $\phi_{\mathrm{s}}=-0.05$ for $\alpha=60^{\circ}$ and $\zeta=42^{\circ}$.

PSR J1702-4310. The top panel of Fig. 19 show the radio polarization data of PSR J1702-4310. The associated RVM 
$\mathrm{J} 1648-4611,1400 \mathrm{MHz}$
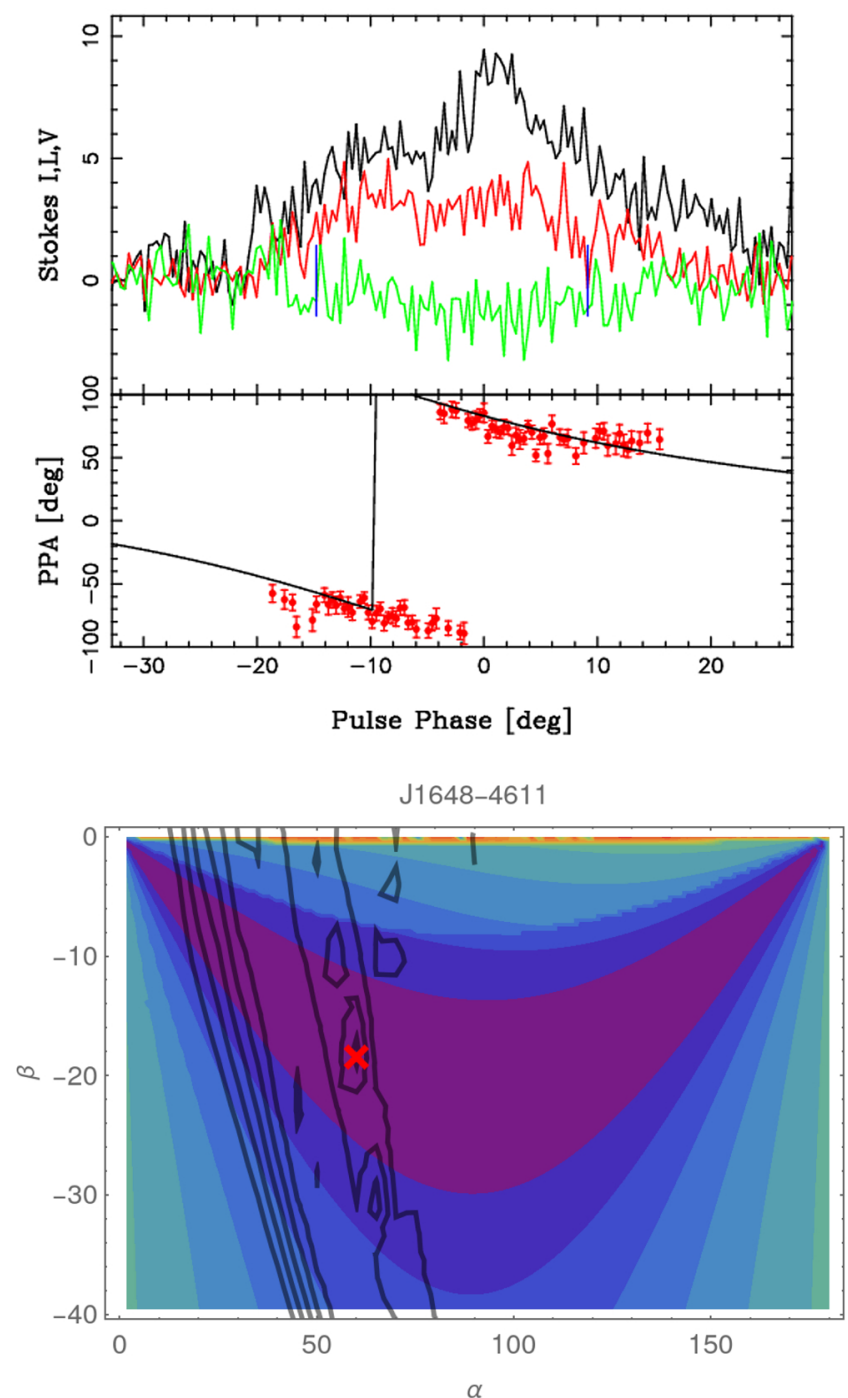

$\alpha$



Fig. 18. Same as Fig. 8, but for J1648-4611.

constraints are given in the middle panel, and are not very constraining. This is another example of $\alpha>90^{\circ}$. One best fitting geometry, indicated by the red cross, produces a single gammaray peak (bottom panel). The phase shift is $\phi_{\mathrm{s}}=-0.05$ for $\alpha=25^{\circ}$ and $\zeta=32^{\circ}$. We do not expect this fit to be very reli-
J1702-4128, $1400 \mathrm{MHz}$

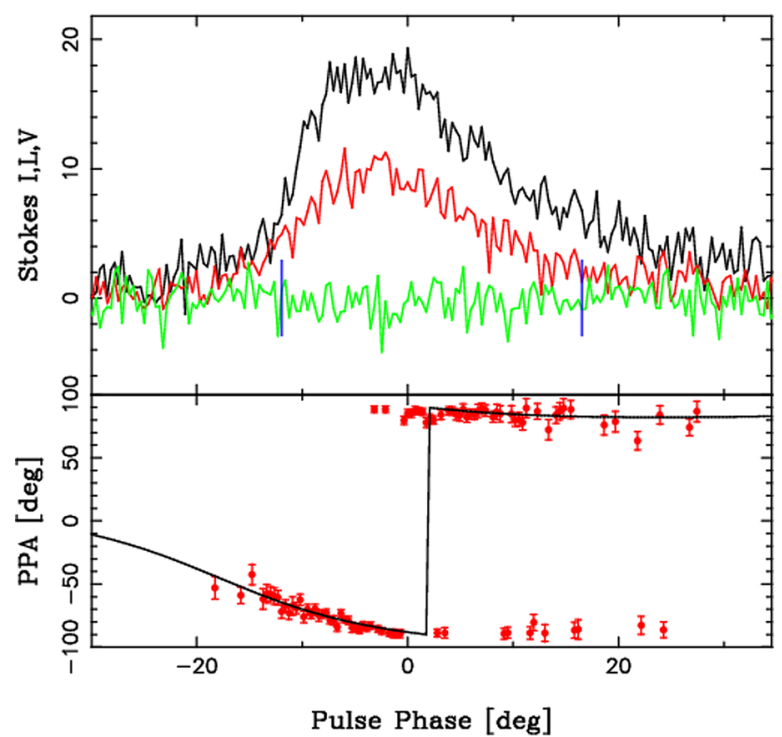

J1702-4128

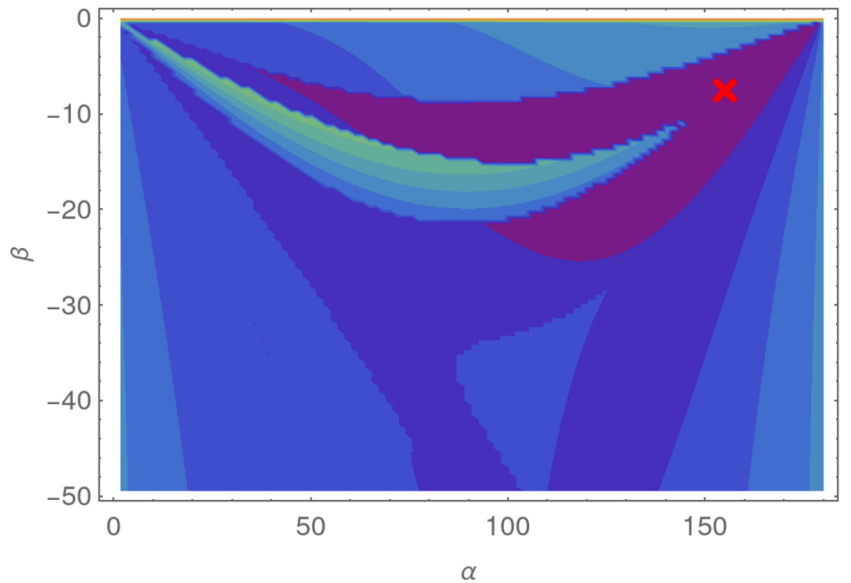

$\alpha$



Fig. 19. J1702-4310 $\log \chi^{2}$ contour plots for radio polarization fits (in colour) and for gamma-ray light curves (solid black lines).

able because the gamma-ray statistics are weak. Switching back to the real geometry, we get $\alpha=155^{\circ}$ and $\zeta=148^{\circ}$.

PSR J1709-4429. Very good PPA data are available for this pulsar (top panel of Fig. 20). Here too, one geometrical configuration is highlighted, coincident with both radio and gamma-rays 
$\mathrm{J} 1709-4429,1400 \mathrm{MHz}$

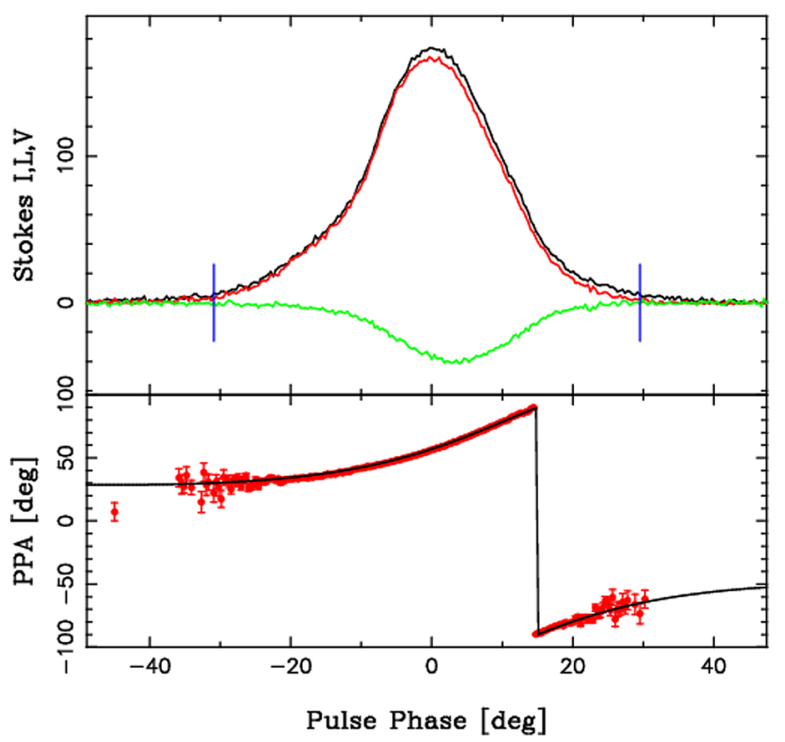

$J 1709-4429$

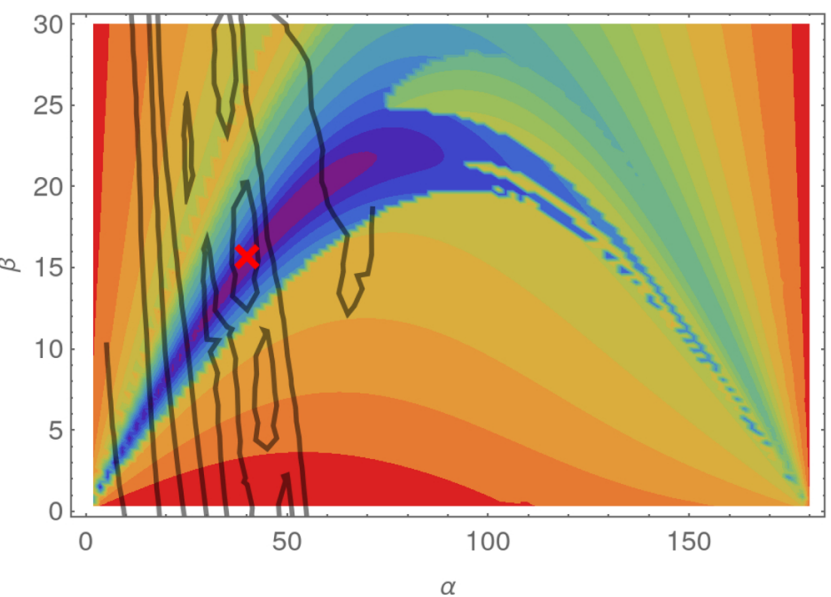

J1709-4429 $\alpha=40^{\circ} ; \zeta=56^{\circ} ; \phi_{S}=-0.1$

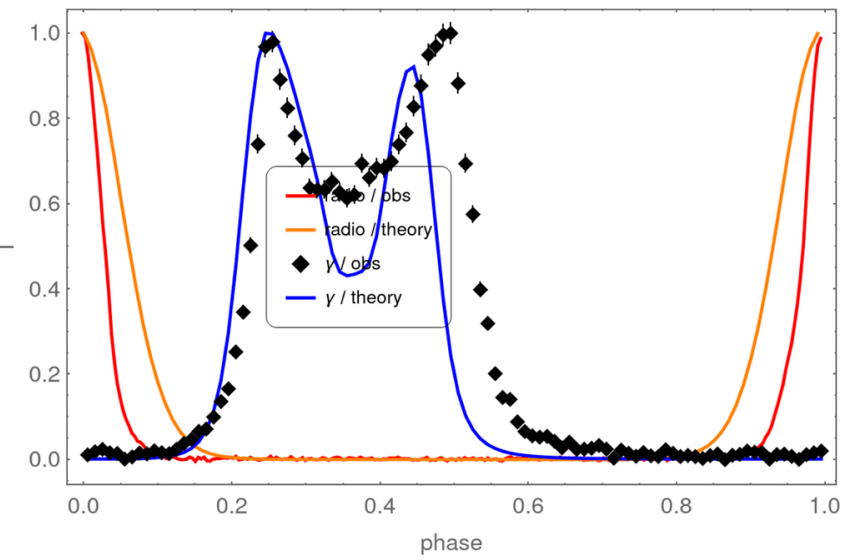

Fig. 20. Same as Fig. 8, but for J1709-4429.

(middle panel). It produces a double-peaked gamma-ray light curve (bottom panel). The phase shift is $\phi_{\mathrm{s}}=-0.1$ for $\alpha=40^{\circ}$ and $\zeta=56^{\circ}$.

PSR J1718-3825. In the same vein as for the previous pulsar, the top panel of Fig. 21 show the PPA evolution leading to a well-defined radio-constrained geometry (middle panel) pick-
$\mathrm{J} 1718-3825,1400 \mathrm{MHz}$

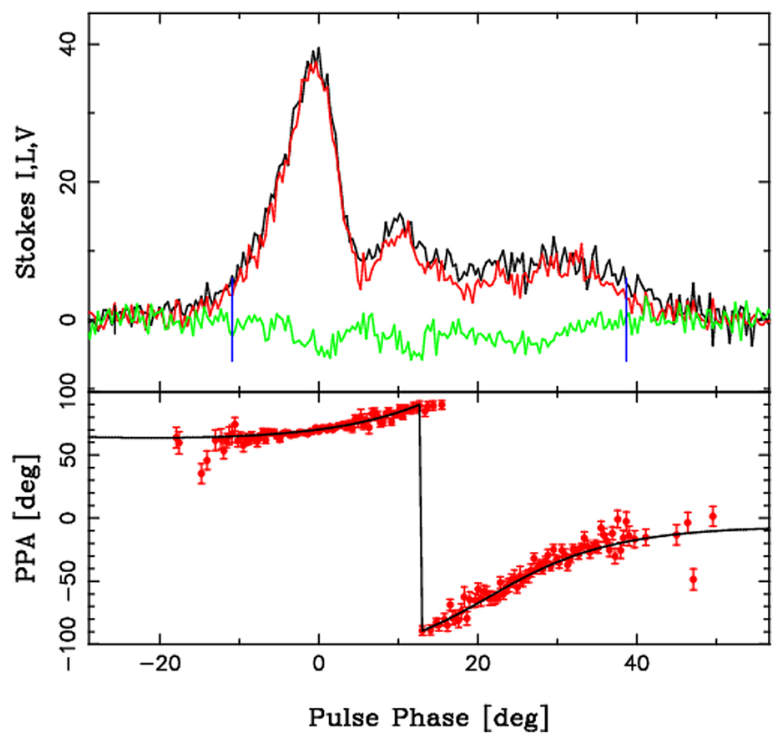

J1718-3825

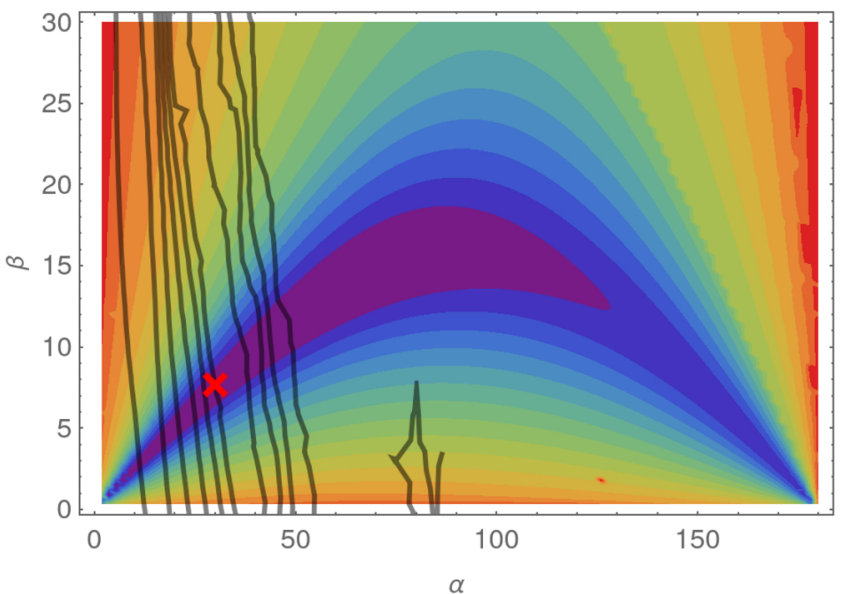

$\mathrm{J} 1718-3825 \alpha=30^{\circ} ; \zeta=38^{\circ} ; \phi_{S}=-0.06$

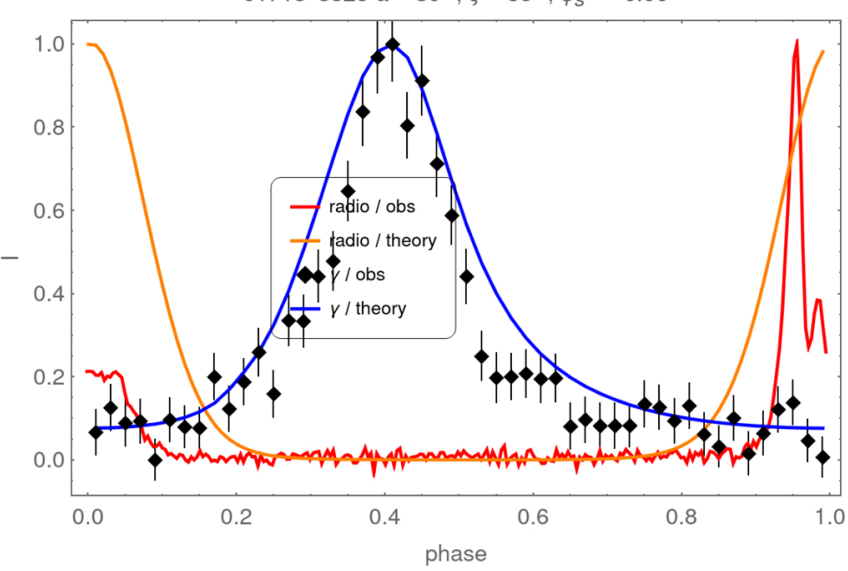

Fig. 21. Same as Fig. 8, but for J1718-3825.

ing out one configuration with the red cross coincident with both wavelengths, producing a single-peaked gamma-ray profile. The phase shift is $\phi_{\mathrm{s}}=-0.06$ for $\alpha=30^{\circ}$ and $\zeta=38^{\circ}$.

PSR J1801-2451. This pulsar shows a strongly doublepeaked gamma-ray profile with one radio pulse (bottom panel 
of Fig. 22). The good radio PPA data (top panel) furnish a reasonable constraint on the geometry (middle panel). The best fit is nearly an orthogonal rotator with a line of sight almost located in the equatorial plane. The phase shift is $\phi_{\mathrm{s}}=-0.12$ for $\alpha=85^{\circ}$ and $\zeta=72^{\circ}$. This configuration shows a second weak radio peak, the interpulse, not seen in the data. We therefore conclude that the true geometry must slightly deviate from our configuration, especially because we do not model the radio emission cone. This second pulse would disappear if a smaller cone of emission is used.

PSR J1835-1106. The radio data of this pulsar are shown in the top panel of Fig. 23. The RVM constraints are well defined in the middle panel. We found a best fit with the red cross producing only one gamma-ray peak with a phase shift of $\phi_{\mathrm{s}}=0.03$ for $\alpha=30^{\circ}$ and $\zeta=36^{\circ}$. The second gamma-ray pulsar catalogue reports the presence of two peaks. Therefore here again, the joined radio gamma-ray constraint seems to lead to some inconsistency with the data. These discrepancies must be carefully analysed, but we are waiting for better observations from Fermi/LAT, supposed to be published in a third gamma-ray pulsar catalogue, before exploring the implications for the emission mechanism.

PSR J2240+5832. This is the last example in our sample showing good radio PPA data (top panel of Fig. 24) (Theureau et al. 2011). The associated RVM constraints are good (middle panel); the radio gamma-ray overlapping region leading to the best geometry is depicted by the red cross. The corresponding gamma-ray light curve is shown in the bottom panel for a phase shift of $\phi_{\mathrm{s}}=-0.09$ with $\alpha=60^{\circ}$ and $\zeta=80^{\circ}$. The weak radio interpulse is predicted, but due to the large opening of the emission cone. According to the narrow width of the radio pulse, this emission cone is largely overestimated and should disappear when reduced to the real size of the radio observations (red solid line).

\subsection{Only gamma-ray fits}

The second part of the sample includes only gamma-ray pulsars not showing a radio signal loud enough for performing a reasonable RVM fit, as done in the previous section. Nevertheless, gamma-ray light curve fitting alone can already help to constrain the geometry of many individual pulsars. Below, we summarize the best fit for some of these young radio gamma-ray pulsars. Figure 25 gives an overview of our fitting results.

PSR JJ0248+6021. It is a single-peaked radio and gammaray pulsar. By only fitting the gamma-ray light curve and its delay compared to the radio profile, we arrive at the best geometry given by a phase shift of $\phi_{\mathrm{s}}=-0.07$ for $\alpha=30^{\circ}$ and $\zeta=42^{\circ}$. Some other very similar angles also give reasonable fits, but they are not shown.

PSR J1016-5857. This pulsar shows two sharp gamma-ray peaks separated by a kind of bridge emission. We are able to fit these two peaks, but not the bridge emission. The phase shift of $\phi_{\mathrm{s}}=-0.02$ for $\alpha=40^{\circ}$ and $\zeta=64^{\circ}$.

PSR J1019-5749. The radio pulse of this pulsar spans almost the entire period, but this is certainly an artefact due to its large dispersion measure. Having also only one gamma-ray peak, we indeed found a small obliquity of $\alpha \approx 30^{\circ}$ with $\zeta=42^{\circ}$ and an additional offset of $\phi_{\mathrm{s}}=0.07$. Larger obliquities are also permissible with slightly less good fits. They are not shown.
$\mathrm{J} 1801-2451,1400 \mathrm{MHz}$
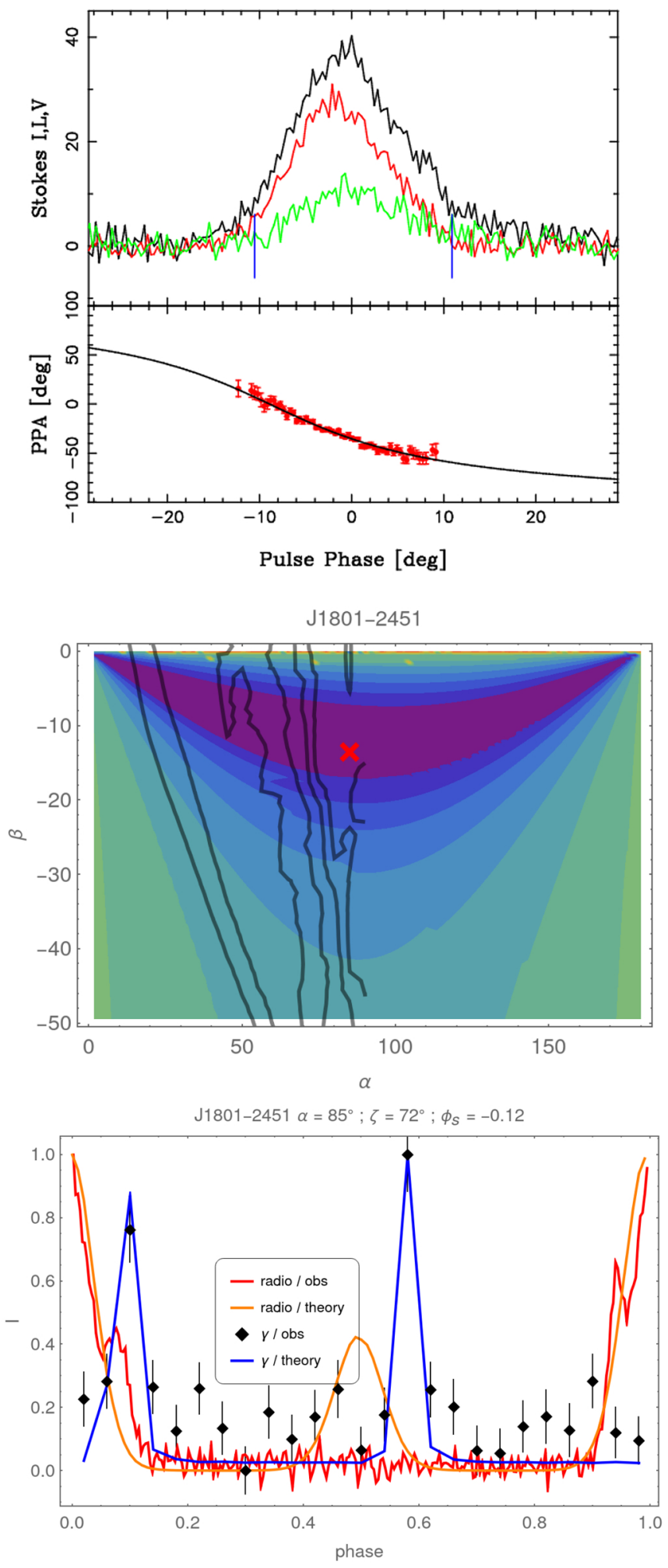

Fig. 22. Same as Fig. 8, but for J1801-2451.

PSR J1028-5819. This pulsar shows one radio pulse and two narrow strongly peaked gamma-ray pulses. The peak separation and shape are well fitted by the striped wind model with $\alpha \approx 75^{\circ}$ and $\zeta=64^{\circ}$ with a small offset of $\phi_{\mathrm{s}}=-0.01$. 
J1835-1106, $1400 \mathrm{MHz}$


Fig. 23. Same as Fig. 8, but for J1835-1106.

PSR J1509-5850. Similar to the previous case, but without interpulse emission, PSR J1509-5850 shows two gammaray peaks that are not well separated. The best fit is associated with a phase shift of $\phi_{\mathrm{s}}=-0.06$ for $\alpha=40^{\circ}$ and $\zeta=24^{\circ}$. We did not find any better geometry reproducing two unresolved gamma-ray peaks.
$\mathrm{J} 2240+5832,1400 \mathrm{MHz}$

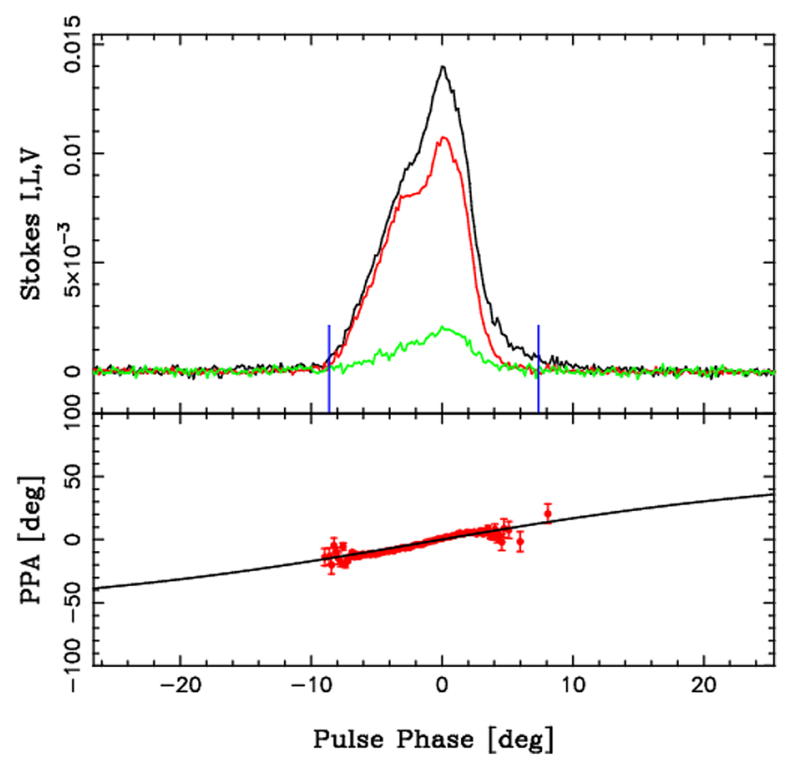

$\mathrm{J} 2240+5832$

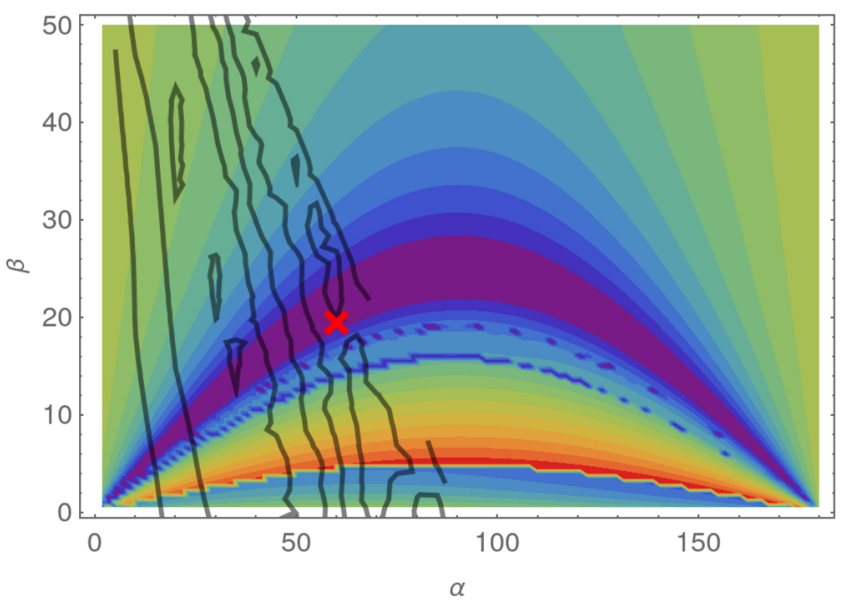

$\mathrm{J} 2240+5832 \alpha=60^{\circ} ; \zeta=80^{\circ} ; \phi_{S}=-0.09$

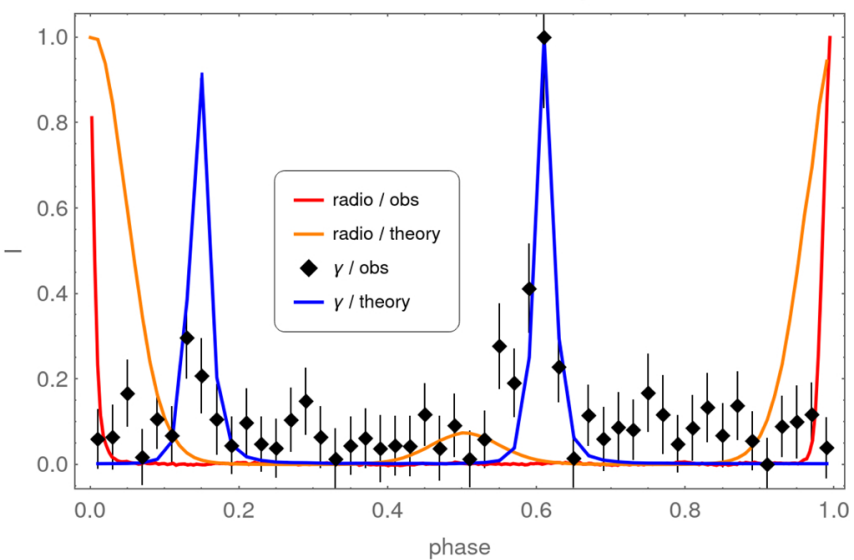

Fig. 24. Same as Fig. 8, but for J2240+5832.

PSR J1730-3350. This is a single radio and gamma-ray pulse profile pulsar. Best fitting parameters are a phase shift of $\phi_{\mathrm{s}}=-0.05$ for $\alpha=20^{\circ}$ and $\zeta=36^{\circ}$.

PSR J1747-2958. This pulsar is similar to PSR J10165857 , showing the same radio and gamma-ray profiles with a 



Fig. 25. Best fit parameters and gamma-ray light curves for the second part of the young radio-loud gamma-ray pulsar sample not having usable RVM fits.

weak bridge emission. Its fitting parameters are therefore close to the one used for PSR J1016-5857 with a phase shift of $\phi_{\mathrm{s}}=-0.07$ for $\alpha=45^{\circ}$ and $\zeta=68^{\circ}$.

PSR J1907+0602. This pulsar has two separated gammaray peaks with a bridge emission and very noisy radio pulse, possibly with an interpulse emission. Our best fit sets $\alpha=45^{\circ}$ and $\zeta=68^{\circ}$ and the phase shift to $\phi_{\mathrm{s}}=-0.04$. Neverthe- less, from the radio interpulse we would expect an orthogonal rotator.

PSR J1952+3252. This pulsar has two well separated but asymmetrical gamma-ray peaks and one radio pulse. The asymmetry cannot be explained by our symmetrical striped wind model. The parameters used in the plot are a phase shift of $\phi_{\mathrm{s}}=-0.06$ for $\alpha=60^{\circ}$ and $\zeta=84^{\circ}$. 


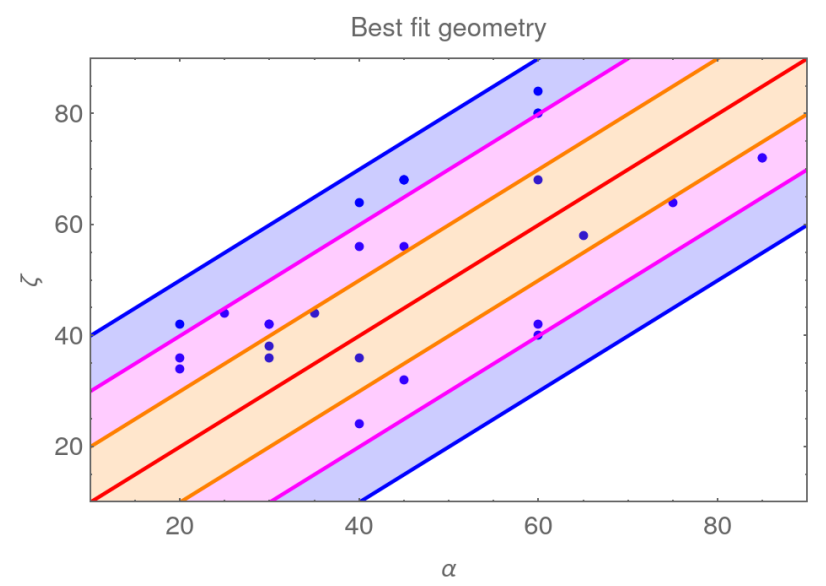

Fig. 26. Correlation between the angles $\alpha$ and $\zeta$ for our best fits (blue dots, see Table 2). The red line shows $\alpha=\zeta$, the orange lines are offset by $\pm 10^{\circ}$, the magenta lines by $\pm 20^{\circ}$, and the blue lines by $\pm 30^{\circ}$.

PSR J2021+3651. This is again an interesting example of prominent and symmetric gamma-ray pulse profiles and a clear single radio pulse. The best fit parameters are a phase shift of $\phi_{\mathrm{s}}=-0.09$ for $\alpha=60^{\circ}$ and $\zeta=80^{\circ}$.

$P S R$ J2030+3641. This is a very noisy radio and gammaray pulsar. It has been fitted by a single gamma-ray profile such that the phase shift is $\phi_{\mathrm{s}}=-0.07$ for $\alpha=20^{\circ}$ and $\zeta=42^{\circ}$.

PSR J2032+4127. Another example of two narrow gammaray peaks with a single radio pulse. It has been fitted by an almost orthogonal rotator with a phase shift of $\phi_{\mathrm{s}}=-0.08$ for $\alpha=85^{\circ}$ and $\zeta=72^{\circ}$.

PSR J2043+2740. A noisy gamma-ray pulsar with two pronounced gamma-ray pulses and bridge emission. The phase shift is $\phi_{\mathrm{s}}=-0.06$ for $\alpha=45^{\circ}$ and $\zeta=68^{\circ}$.

PSR J2229+6114. This single gamma-ray peak pulsar with asymmetrical leading and trailing edge has been fitted with a phase shift of $\phi_{\mathrm{s}}=-0.03$ for $\alpha=35^{\circ}$ and $\zeta=44^{\circ}$.

\subsection{Summary}

Gathering all the results from the two previous sections, our best fit values for the angles $\alpha$ and $\zeta$ and for the phase offset $\phi_{\mathrm{s}}$ are summarized in Table 2.

Figure 26 summarizes the best fit angles $\alpha$ and $\zeta$, showing that they follow the relation $|\zeta-\alpha| \lesssim 30^{\circ}$, which is slightly larger than the value we expected from the constraints in Sect. 3.2. This means that according to our model, some pulsars could have an emission height above the fiducial altitude of $0.05 r_{\mathrm{L}}$. Jointly, the distribution of angles $\alpha, \beta$, and offsets $\phi_{\mathrm{s}}$ according to the same best fit values are shown in the histograms of Fig. 27. Half of the sample has an obliquity of less than $45^{\circ}$. The line of sight angle $\beta$ is distributed approximately symmetrically with respect to the magnetic axis. Interestingly, we found an important cluster of offsets around a phase $\phi_{\mathrm{s}} \approx-0.05$ equitably distributed on both sides of this value with some marginal outliers lying more than 0.15 away from this median value of -0.05 . This clustering indicates that some systematics have not been included in our study. The first gamma-ray peak is expected to come earlier than predicted by our model. One possibility would be that the striped wind emission is delayed, not peaking right at the light cylinder
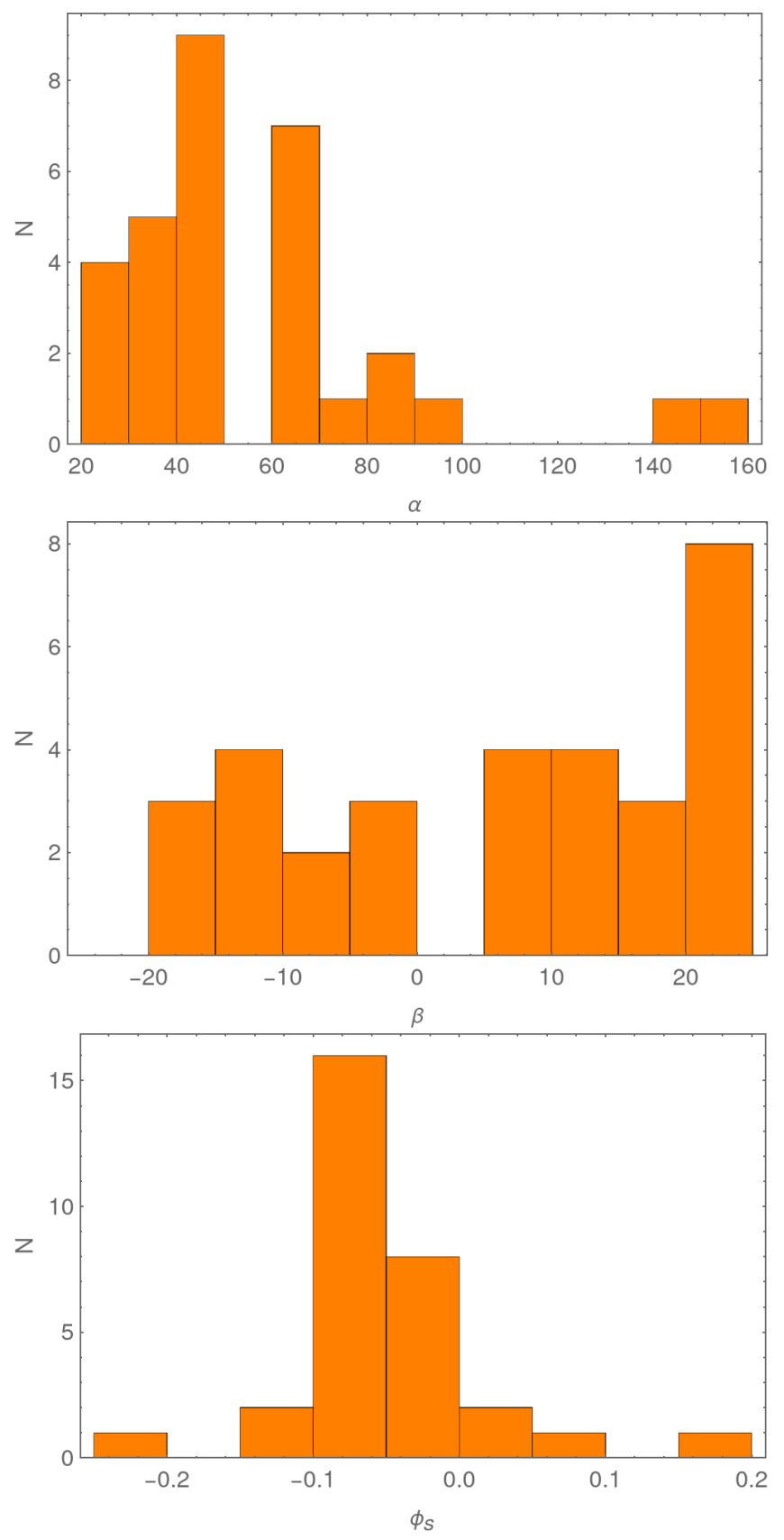

Fig. 27. Histogram of best fit parameters given by the obliquity $\alpha$ the line of sight $\beta$ and the phase offset $\phi_{\mathrm{s}}$ for the best fits given in Table 2 .

but at larger distances, a fraction of a light cylinder radii $\Delta r$ away from the light cylinder. This repelling to larger distances automatically shifts the gamma-ray profile closer to the radio pulse by a phase $\phi \approx \frac{\Delta r}{2 \pi r_{\mathrm{L}}}$, where $\Delta r$ measures this additional distance. Setting $\Delta r \approx r_{\mathrm{L}} / 2$ leads to an additional phase shift of $\phi \approx 0.08$, sufficient to explain the histogram. Another possibility would be the forward beaming of the current sheet emission at the light cylinder, forward with respect to the rotation direction, due to an azimuthal velocity close to the speed of light in this region. These aberration effects also shorten the time lag between radio and gamma rays. Some additional work is needed to accurately pin down the geometry. Only careful individual pulsar analysis will be able to tune these parameters firmly to irrelevant uncertainties. 


\section{Conclusions}

Multi-wavelength observations of neutron star pulsed emission offers a precious tool to explore the emission location within the pulsar magnetosphere and wind. We showed that simultaneously fitting the radio and gamma-ray pulse profile of radioloud gamma-ray pulsars severely constrains the geometry of the dipole magnetic field and observer line of sight with respect to the rotation axis. Moreover, when radio polarization data are available, additional constraints arise from fitting the rotating vector model. We showed that the RVM alone cannot be used to constrain the geometry of radio pulsar simply by minimizing the RVM $\chi^{2}$ fit. Some additional knowledge from other wavelengths is required. In most cases, the gamma-ray and radio fitting regions possess an overlapping area consistent with the gamma-ray light curves, reducing the uncertainties on obliquity and line of sight. We applied our method to a large sample of radio-loud gamma-ray pulsars with small error boxes, except for rare cases. As a good proxy, radio emission emanates from altitudes around $0.05-0.1 r_{\mathrm{L}}$, where the magnetic field is dominantly dipolar, whereas gamma-ray photons are produced at the light cylinder or slightly further away, extending to several $r_{\mathrm{L}}$ around the striped wind current sheet.

From a theoretical point of view, this study was only based on the impact of geometrical considerations on the radio and light curve association, in the limit of a force-free magnetosphere. There is no mention of either energetic or particle dynamics. Particle acceleration and radiation needs to go beyond the force-free approximation by adding some dissipation, such as resistivity or radiation reaction damping. When acceleration and radiation sites have been accurately localized by these selfconsistent models, we will be able to produce multi-wavelength phase-resolved spectra and light curves to pin down even better and more faithfully the magnetosphere geometry and its internal electrodynamics. Observational signatures of this dissipative magnetosphere needs to be performed to further support our emission model.

From an observational point of view, some pulsars would greatly benefit from better signal-to-noise ratios of the radio and gamma-ray pulse profiles. The upcoming third pulsar catalogue in gamma rays and the construction of the square kilometer array promise to take a big step towards our understanding of pulsar emission mechanisms.
Acknowledgements. We are grateful to the referee for helpful comments and suggestions. This work has been supported by the CEFIPRA grant IFC/F5904B/2018 and ANR-20-CE31-0010. We acknowledge the High Performance Computing Center of the University of Strasbourg for supporting this work by providing scientific support and access to computing resources. We thank Lucas Guillemot for sending us some radio polarization data and David Smith for stimulating discussions. DM acknowledges the support of the Department of Atomic Energy, Government of India, under project no. 12-R\&D-TFR-5.02-0700.

\section{References}

Abdo, A. A., Ackermann, M., Ajello, M., et al. 2010, ApJS, 187, 460 Abdo, A. A., Ajello, M., Allafort, A., et al. 2013, ApJS, 208, 17 Benli, O., Pétri, J., \& Mitra, D. 2021, A\&A, 647, A101

Blaskiewicz, M., Cordes, J. M., \& Wasserman, I. 1991, ApJ, 370, 643 Bogovalov, S. V. 1999, A\&A, 349, 1017

Cao, G., Zhang, L., \& Sun, S. 2016a, MNRAS, 455, 4267

Cao, G., Zhang, L., \& Sun, S. 2016b, MNRAS, 461, 1068

Cerutti, B., Philippov, A., Parfrey, K., \& Spitkovsky, A. 2015, MNRAS, 448, 606

Contopoulos, I., Kazanas, D., \& Fendt, C. 1999, ApJ, 511, 351

Deutsch, A. J. 1955, Ann. Astrophys., 18, 1

Everett, J. E., \& Weisberg, J. M. 2001, ApJ, 553, 341

Johnston, S., \& Kerr, M. 2018, MNRAS, 474, 4629

Kalapotharakos, C., Contopoulos, I., \& Kazanas, D. 2012, MNRAS, 420, 2793 Kalapotharakos, C., Harding, A. K., Kazanas, D., \& Brambilla, G. 2017, ApJ, 842,80

Komissarov, S. S. 2006, MNRAS, 367, 19

Kramer, M., \& Johnston, S. 2008, MNRAS, 390, 87

Li, J., Spitkovsky, A., \& Tchekhovskoy, A. 2012, ApJ, 746, 60

Michel, F. C. 1973, ApJ, 180, L133

Mitra, D. 2017, J Astrophys. Astron., 38, 52

Mitra, D., \& Li, X. H. 2004, A\&A, 421, 215

Mitra, D., \& Rankin, J. M. 2011, ApJ, 727, 92

Mitra, D., Rankin, J. M., \& Gupta, Y. 2007, MNRAS, 379, 932

Mitra, D., \& Seiradakis, J. H. 2004, ArXiv e-prints [arXiv:astro-ph/0401335]

Parfrey, K., Beloborodov, A. M., \& Hui, L. 2012, MNRAS, 423, 1416

Phillips, J. A. 1992, ApJ, 385, 282

Pierbattista, M., Harding, A. K., Grenier, I. A., et al. 2015, A\&A, 575, A3

Pierbattista, M., Harding, A. K., Gonthier, P. L., \& Grenier, I. A. 2016, A\&A, 588, A137

Pétri, J. 2011, MNRAS, 412, 1870

Pétri, J. 2012, MNRAS, 424, 605

Pétri, J. 2018, MNRAS, 477, 1035

Radhakrishnan, V., \& Cooke, D. J. 1969, Astrophys. Lett., 3, 225

Rookyard, S. C., Weltevrede, P., \& Johnston, S. 2015, MNRAS, 446, 3356

Seyffert, A. S., Venter, C., de Jager, O. C., \& Harding, A. K. 2011, ArXiv e-prints [arXiv:1105.4094]

Spitkovsky, A. 2006, ApJ, 648, L51

Theureau, G., Parent, D., Cognard, I., et al. 2011, A\&A, 525, A94

Watters, K. P., Romani, R. W., Weltevrede, P., \& Johnston, S. 2009, ApJ, 695, 1289

Weltevrede, P., \& Johnston, S. 2008, MNRAS, 391, 1210

Weltevrede, P., \& Wright, G. 2009, MNRAS, 395, 2117 